\title{
Cdk5/p25-Induced Cytosolic PLA2-Mediated Lysophosphatidylcholine Production Regulates Neuroinflammation and Triggers Neurodegeneration
}

\author{
Jeyapriya R. Sundaram, ${ }^{1,2}$ Elizabeth S. Chan, ${ }^{1,3}$ Charlene P. Poore, ${ }^{1,3}$ Tej K. Pareek, ${ }^{6}$ Wei Fun Cheong, ${ }^{1,3}$ \\ Guanghou Shui, ${ }^{1,3}$ Ning Tang, ${ }^{1,2}$ Chian-Ming Low, ${ }^{1,2,5}$ Markus R. Wenk, ${ }^{1,3,4}$ and Sashi Kesavapany ${ }^{1,3}$ \\ ${ }^{1}$ Neurobiology and Ageing Program, Centre for Life Sciences, Yong Loo Lin School of Medicine, and Departments of ${ }^{2}$ Pharmacology, ${ }^{3}$ Biochemistry, \\ ${ }^{4}$ Biological Sciences, and ${ }^{5}$ Anesthesia, National University of Singapore, Singapore 117456, and ${ }^{6}$ Department of Pediatrics, Case Western Reserve \\ University, Cleveland, Ohio 44106
}

The deregulation of cyclin-dependent kinase 5 (Cdk5) by p 25 has been shown to contribute to the pathogenesis in a number of neurodegenerative diseases such as amyotrophic lateral sclerosis (ALS), Parkinson's disease (PD) and Alzheimer's disease (AD). In particular, p25/Cdk5 has been shown to produce hyperphosphorylated tau, neurofibrillary tangles as well as aberrant amyloid precursor protein processing found in AD. Neuroinflammation has been observed alongside the pathogenic process in these neurodegenerative diseases, however the precise mechanism behind the induction of neuroinflammation and the significance in the AD pathogenesis has not been fully elucidated. In this report, we uncover a novel pathway for p25-induced neuroinflammation where p 25 expression induces an early trigger of neuroinflammation in vivo in mice. Lipidomic mass spectrometry, in vitro coculture and conditioned media transfer experiments show that the soluble lipid mediator lysophosphatidylcholine (LPC) is released by p 25 overexpressing neurons to initiate astrogliosis, neuroinflammation and subsequent neurodegeneration. Reverse transcriptase PCR and gene silencing experiments show that cytosolic phospholipase 2 (cPLA2) is the key enzyme mediating the p25-induced LPC production and cPLA2 upregulation is critical in triggering the p25-mediated inflammatory and neurodegenerative process. Together, our findings delineate a potential therapeutic target for the reduction of neuroinflammation in neurodegenerative diseases including AD.

\section{Introduction}

Neurodegenerative diseases are characterized by a progressive loss of neurons in the CNS, in which affected neurons often display characteristic hallmarks of a particular disease process. Alzheimer's disease $(\mathrm{AD})$ is characterized by the deposition of $\beta$-amyloid plaques, accumulation of phospho-tau containing neurofibrillary tangles, reactive astrogliosis and loss of neurons and synapses in selected areas of the brain (Trojanowski et al., 1993). Cdk5, a member of the cyclin-dependent kinase (Cdk) family of Ser/Thr kinases, is a master regulator of the cytoarchitecture of the brain (Meyerson et al., 1991). The Cdk5 activator protein, p35 can be cleaved to a smaller fragment p25, by the calcium activated protease, calpain. Cdk5/p25 has a mislocalized activity as well as a longer half-life (Patrick et al., 1999). Increased levels of p25 protein have also been found in the postmortem

Received Oct. 14, 2011; revised Nov. 16, 2011; accepted Nov. 22, 2011.

Author contributions: T.K.P., C.-M.L., M.R.W., and S.K. designed research; J.R.S., E.S.C., C.P.P., T.K.P., W.F.C., and N.T. performed research; J.R.S., E.S.C., T.K.P., G.S., and S.K. analyzed data; J.R.S. and S.K. wrote the paper.

This work was supported by National Medical Research Council Grants WBS 183-000-179-213 and 184-000-180213, and Singapore National Research Foundation Competitive Research Programme Award No. 2007-04. We thank Dr. Paul MacAry, Immunology Program, Life Sciences Institute, National University of Singapore, for the kind gift of antibodies CD4 and CD8. We also thank Noor Hazim Bin Sulaimee for technical assistance.

Correspondence should be addressed to Dr. Sashi Kesavapany, National University of Singapore, 28 Medical Drive, \# 04-21, Singapore 117456. E-mail: sashi.s.kesavapany@gsk.com.

DOI:10.1523/JNEUROSCI.5177-11.2012

Copyright $\odot 2012$ the authors $\quad 0270-6474 / 12 / 321020-15 \$ 15.00 / 0$ brain tissue of $\mathrm{AD}$ patients (Patrick et al., 1999; Tseng et al., 2002). Although this has been debated by a number of groups (Takashima et al., 2001; Bian et al., 2002), the proteolytic cleavage of p35 to p25, which has been shown to hyperactivate Cdk5 is without doubt. Deregulation of Cdk5 activity, accompanied by the accumulation of $\mathrm{p} 25$ has been implicated as a causative factor in the pathogenesis of neurodegenerative diseases (Nguyen et al., 2001; Smith et al., 2006). Although few studies debated the involvement of p25/Cdk5 in neurodegeneration (Hallows et al., 2006), large bodies of evidence show the involvement of p25/ Cdk5 hyperactivation in the development of pathological hallmarks of AD (Otth et al., 2002; Town et al., 2002; Cruz et al., 2003; Noble et al., 2003; Lopes et al., 2007; Saito et al., 2007).

Numerous neurodegenerative diseases share the common feature of neuroinflammation, which is a complex cascade of selfdefense responses to injurious stimuli in the CNS. Increasing evidence has confirmed a major role of reactive astrocytes and microglia in the initiation and exacerbation of CNS inflammation (Markiewicz and Lukomska, 2006). Although, recent report suggests that there is a close association between neurodegeneration and p25-mediated neuroinflammation, the precise mechanism behind the link is not fully described (Muyllaert et al., 2008). Hence our study aims to investigate the mechanism behind the early events that trigger the cellular alterations in neuroinflammation leading to neurodegeneration in p25-expressing neurons. 
In this report, we have elucidated a pathway of p25-induced neuroinflammation where p25 expression, at very early time points, induces neuroinflammation in vitro and in vivo in the absence of any amyloid or tau pathology. We show that p25 overexpressing neurons release a soluble lipid factor, lysophosphatidylcholine (LPC), through the upregulation of cytosolic PLA2 (cPLA2) which causes astrogliosis and increased proinflammatory cytokine production. We also show that the inflammatory component produced during p 25 overexpression could trigger later pathological changes in the neurodegenerative process. Our report unveils cPLA2 as a potential therapeutic target in p25mediated neuroinflammation and neurodegeneration.

\section{Materials and Methods}

Animal handling. All animal experimentation was performed according to approved protocols by the Institutional Animal Care and Use Committee of the National University of Singapore.

Antibodies. Antibodies used for Western blot analyses were rabbit antiGFAP (Sigma, 1:1000), rabbit C-8 (Santa Cruz Biotechnology, 1:500), mouse monoclonal anti-cPLA2 (Santa Cruz Biotechnology, 1:200) and mouse anti- $\alpha$-tubulin (Sigma, 1:10,000). Secondary horseradish peroxidase-conjugated antibodies (GE Healthcare) were used at 1:1000 dilutions. Antibodies used for immunohistochemistry were mouse antiGFAP (Sigma, 1:1000), mouse monoclonal AT8 (Pierce, 1:100), anti- $\beta$ amyloid 1-42 (Millipore, 1:100), anti-CD11b (Millipore, 1:200), rabbit anti-mouse tPA (Molecular Innovations, 1:500), mouse anti-CD4 and anti-CD8 (BioLegend, 1:200). Secondary fluorescence-conjugated antibodies Alexa Fluor 488 and Alexa Fluor 594 (Invitrogen) were used at 1:200 dilutions.

p25 transgenic mouse model. p25 transgenic (p25Tg) mice C57BL/6-Tg (tetO-CDK5R1/GFP) 337Lht/J (The Jackson Laboratory) were mated with Camk2a transgenic mice B6; CBA-Tg (Camk2a-tTA) 1Mmay/J (The Jackson Laboratory) to generate bitransgenic offspring that inducibly overexpress human p25 gene under the control of the tetracyclinederivative, doxycycline. These mice were initially described by Cruz et al. (2003). All mice in this study were conceived and raised in the presence of doxycycline (Sigma; $200 \mu \mathrm{g} / \mathrm{ml}$ ) in drinking water, for 6 weeks postnatal to avert any potential developmental consequences from the expression of $\mathrm{p} 25$. The expression of $\mathrm{p} 25$ was induced by removal of doxycycline and brain samples were collected at different weeks of induction periods. Littermates of either sex mice were used for the experiments. Same sex mice were used for comparison whenever possible.

Mammalian cell culture. Primary mouse cortical neurons were cultured as described previously (Kesavapany et al., 2004b). Briefly, mouse embryonic day 16-18 (E16-E18) cortical neurons were cultured in Neurobasal medium with B27 supplement (Invitrogen) containing 100 $\mathrm{IU} / \mathrm{ml}$ penicillin, $100 \mu \mathrm{g} / \mathrm{ml}$ streptomycin, and $2 \mathrm{~mm}$ glutamine. Primary glial cultures (mixed glial culture) using P0-P2 pups followed the identical procedure and were cultured in DMEM supplemented with $2 \mathrm{~mm}$ penicillin streptomycin glutamine, $10 \%$ FBS and 10 mm sodium pyruvate at $37^{\circ} \mathrm{C}$ with $5 \% \mathrm{CO}_{2}$. Human embryonic kidney (HEK) FT cell line was obtained from the American Type Culture Collection and cultured in DMEM supplemented with $2 \mathrm{~mm}$ penicillin streptomycin glutamine, $10 \% \mathrm{FBS}$, and $1.5 \mu \mathrm{g} / \mathrm{ml}$ sodium bicarbonate at $37^{\circ} \mathrm{C}$ with $5 \% \mathrm{CO}_{2}$.

Lentivirus production and transduction. Lentiviruses of empty vector (EV) and p25-EGFP were prepared as described previously (Zheng et al., 2005). Briefly, HEK293-FT cells were co-transfected with p25-LV or EV-LV and Virapower Packaging Mix using Lipofectamine 2000 and following the methods described in the ViraPower Lentiviral Expression system (Invitrogen). Cell-free viral supernatants were collected after 48 and $72 \mathrm{~h}$ and filtered through a $0.45 \mu \mathrm{m}$ filter and then concentrated $\sim 40$-fold by ultracentrifugation through Amicon Ultra-15 centrifugal filter units with Ultracel-100 membranes (Millipore). Viral titers, expressed as the percentage of total cells expressing the p25-GFP/EV-GFP $(\sim 80 \%)$, were determined by transduction of 7 days in culture (DIC) cortical neurons and GFP-fluorescence was visualized after $72 \mathrm{~h}$.

Coculture and supernatant transfer experiments. EV-LV/p25-LVtransduced cortical neurons or $5 \mathrm{~d}$ induced $\mathrm{p} 25 \mathrm{Tg} /$ control neurons were cultured on $15 \mathrm{~mm}$ poly-L-lysine-coated coverslips and placed in $3.0 \mu \mathrm{m}$ tissue culture inserts (Greiner bio-one) and glia were plated either on coverslips $(18 \mathrm{~mm})$ or at the bottom of 6 well plates and incubated for $48 \mathrm{~h}$. Alternatively, supernatants from EV-LV/p25-LV virus-transduced cortical neurons or $5 \mathrm{~d}$ induced $\mathrm{p} 25 \mathrm{Tg} /$ control mice neurons were collected and centrifuged at $14,000 \times g$ for $15 \mathrm{~min}$. The cell-free supernatants were then transferred onto glia and incubated for $48 \mathrm{~h}$. The glia from coculture or supernatant transfer experiments were fixed for immunocytochemistry or processed for Western blot analyses.

Factor removal experiments. Cell-free supernatants from EV-LV/p25LV-transduced cortical neurons or $5 \mathrm{~d}$ induced $\mathrm{p} 25 \mathrm{Tg} /$ control mice neurons were treated with DNase $(8 \mu \mathrm{g} / \mathrm{ml})$, RNase $(50 \mu \mathrm{g} / \mathrm{ml})$, and Proteinase $\mathrm{K}(50 \mu \mathrm{g} / \mathrm{ml})$ (Sigma) for $60 \mathrm{~min}$ at $37^{\circ} \mathrm{C}$. The reactions were stopped by boiling the supernatants at $95^{\circ} \mathrm{C}$ for $20 \mathrm{~min}$. The enzymetreated supernatants were then cooled down to $37^{\circ} \mathrm{C}$ and transferred to glia. To remove lipids, cell-free supernatants were passed through the solid phase extraction column (SPE-C18 column, Waters) under vacuum and eluted supernatants were transferred to glia. The samples were collected for Western blotting and immunocytochemistry after $48 \mathrm{~h}$.

Immunocytochemical analysis. Glial cells on coverslips (300,000 cell/18 $\mathrm{mm}$ coverslip) were fixed with $4 \%$ formaldehyde for $20 \mathrm{~min}$. The cells were permeabilized with $0.1 \%$ Triton X-100 for 20 min and blocked with $5 \%$ FBS in PBS for 30 min. Cells were incubated with the primary antibody in 5\% FBS/PBS for $1 \mathrm{~h}$ at room temperature. The cells were washed three times with PBS followed by addition of the appropriate secondary antibody for $1 \mathrm{~h}$ at room temperature and nuclei were counterstained with DAPI (Sigma). The coverslips were then washed with PBS and mounted on glass slides in fluorescence mounting medium (Dako). Fluorescent confocal images were captured with a Zeiss LSM-510 laserscanning confocal microscope at $40 \times$ magnification.

Immunohistochemistry. Mice were perfused with 4\% PFA/PBS and $16 \mu \mathrm{m}$ of brain cryosections were permeabilized, blocked and incubated with primary antibody overnight at $4^{\circ} \mathrm{C}$ in PBS with 5\% FBS. Sections were washed in PBS before incubation with secondary antibody for $1 \mathrm{~h}$ at room temperature and nuclei were stained with DAPI. The immunostained sections were mounted on cover glasses in fluorescent mounting medium. Confocal images were taken at $20 \times, 40 \times$, and $63 \times$ magnifications.

TUNEL assay. TUNEL staining was performed according to manufacturer's instructions using In Situ Cell Death Detection Kit, TMR red (Roche).

Western blot analysis. Mouse brain lysates, total cell lysates and soluble cell lysates were prepared as previously described (Kesavapany et al., 2004a). Briefly, Samples were separated on $4-20 \%(w / v)$ polyacrylamide gels (Invitrogen) and transferred to nitrocellulose membranes and probed with primary antibodies overnight. The membranes were then washed in Tris-buffered saline-Tween 20 (TBST) and incubated with horseradish peroxidase-conjugated mouse or rabbit secondary antibodies for followed by three washes with TBST. Blots were developed using enhanced chemiluminescence (GE Healthcare) according to the instructions of the manufacturer.

In vitro kinase assay. In vitro kinase assays to investigate the changes in Cdk5 activity were performed as published previously (Poore et al., 2010).

Inhibitor studies. BEL (0.3 $\mu \mathrm{M}$; Sigma) (iPLA2 inhibitor) or $10 \mu \mathrm{M}$ AACOCF3 (Biomol) (cPLA2 inhibitor) was incubated with neurons transduced with p25-LV. Neurons were fixed after $48 \mathrm{~h}$ and immunocytochemistry was performed using anti-GFAP antibody.

cPLA2 silencing. cPLA2 silencing was performed in neurons by transduction with cPLA2 shRNA lentivirus (Santa Cruz Biotechnology) according to the manufacturer's instructions. Silencing was validated by reverse transcriptase (RT)-PCR and Western blot analyses.

cPLA2 activity assay. cPLA2 activity was determined for the lysates from neurons transduced with EV-LV or p25-LV using cPLA2 activity Assay Kit (Cayman Chemical) according to the manufacturer's instructions. The results were normalized against protein concentration determination by BCA assays (Pierce Biotechnology).

Real-time PCR. Mice brains were homogenized in TRIzol and total RNA was extracted using RNeasy Mini Kit (Qiagen) and then quantified using a NanoDrop Spectrophotometer (Thermo Scientific). cDNA was synthesized 
according to manufacturer's protocol using High capacity cDNA reverse transcriptase kits (Applied Biosystems). Quantitative real time PCR was performed in a 96-well plate using ABI Prism 7900 HT Fast 9 Detection System (Applied Biosystems) as per manufacturer's instructions. The $25 \mu \mathrm{lPCR}$ mix included $2.5 \mu \mathrm{l}$ of $2 \mathrm{ng} / \mu \mathrm{l} \mathrm{cDNA}$ and $22.5 \mu \mathrm{l}$ of Power SYBR Green PCR master mix (Applied Biosystems) with $0.2 \mu \mathrm{M}$ respective primers. The following primers were used: MIP- $1 \alpha$ (5'-TTGAGCCCCGGAACATTC-3'; $5^{\prime}$-GCAGCAAACAGCTTATAGGAGATG- $3^{\prime}$ ), TNF- $\alpha$ (5' -AGCACAGAAAGCATGATCCG-3'; $5^{\prime}$-GGAGTAGACAAGGTACAACC-3'), TGF- $\beta$ ( $5^{\prime}$-CTTTAGGAAGGACCTGGGTT-3'; $5^{\prime}$-CAGGAGCGCACAATCATGTT- $\left.3^{\prime}\right)$, IL-1 $\beta$ ( $5^{\prime}$-ACCTGCTGGTGTGTGACGTTC-3'; $5^{\prime}$-CAGCACGAGGCTTTTTTGTTGT-3'), iPLA2 (5' -TAACCTGAAGCCACCGACTC-3' ${ }^{\prime} ; 5^{\prime}$-TAGTGTTGATCTCTGATATG-3') and cPLA2 (5' CTGCAAGGCCGAGTGACA-3'; 5' -TTCGCCCACTTCTCTGCAA-3'). The Ct values were determined using default threshold settings in the system software.

Lipid extraction from cell culture media. Lipids were extracted from cell culture media/supernatant using a modified protocol described previously (Bremer and Norum, 1982). In brief, lipids were first extracted using $6 \mathrm{ml}$ of 1-butanol by incubating the cell culture media-butanol solution with agitation at $160 \mathrm{rpm}$ for $2 \mathrm{~h}$ at $4^{\circ} \mathrm{C}$. After the break phase, the butanol extract at the upper layer was transferred to clean glass vial and $5 \mathrm{ml}$ of chloroform was then added to perform a second extraction. The chloroform extract at the bottom phase was obtained after break phase and pooled with the butanol extract from the first extraction. The lipid extracts were then dried under a nitrogen stream and kept at $-80^{\circ} \mathrm{C}$.

Lipids analysis using high performance liquid chromatography/mass spectrometry. Separation and quantification of individual lipids was performed using an Agilent 1200 high-performance liquid chromatography (HPLC) system and a 3200 Q-Trap mass spectrometer (Applied Biosystems) (Chan et al., 2008; Shui et al., 2010). The HPLC system is made up of an Agilent 1200 binary pump, an Agilent 1200 thermo sampler and an Agilent 1200 column oven HPLC conditions Luna 3u silica column (inner diameter $150 \times 2.0$ $\mathrm{mm}$ ); mobile phase A (chloroform/methanol/ammonium hydroxide, 89.5: 10:0.5), B (chloroform/methanol/ammonium hydroxide/water, 55:39:0.5: 5.5); flow rate $350 \mu \mathrm{l} / \mathrm{min}$; $5 \% \mathrm{~B}$ for $3 \mathrm{~min}$, then linearly changed to $30 \% \mathrm{~B}$ in $24 \mathrm{~min}$ and maintained for $5 \mathrm{~min}$, and then linearly changed to $70 \% \mathrm{~B}$ in 5 min and maintained for $7 \mathrm{~min}$. Eluents were changed to the original ratio in $5 \mathrm{~min}$ and maintained for $6 \mathrm{~min}$. Multiple reaction monitoring transitions for individual phosphatidylethanolamine (PE), phosphatidylcholine (PC), phosphatidylserine (PS), phosphatidylinositol (PI), ceramide, and sphingomyelin (SM) species were set up for quantitative analysis. Levels of individual lipid levels were quantified using spiked internal standards PC-14:0/14:0, PE14:0-14:0, PS-14:0/14:0, ceramide, C12-SM and LPC 20:0 (4 $\mu \mathrm{M})$, which were obtained from Avanti Polar Lipids. Dioctanoyl phosphatidylinositol PI-8:0/8:0 was used for phosphatidylinositol quantitation (Echelon Biosciences).

Stereotactic injection of lipids into mouse brain. Mice were placed in a stereotactic frame after induction of anesthesia. The vertex area was exposed after midline incision of the scalp, and a small opening was made with a dental drill according to the following coordinates: caudal to bregma $2 \mathrm{~mm}, 2 \mathrm{~mm}$ lateral to the midline and $1.8 \mathrm{~mm}$ from the surface of the cortex. A Hamilton microsyringe was inserted stereotactically through the hole and $1 \mu \mathrm{l}$ of solution containing lipids $(2 \mu \mathrm{M})$ was injected at a constant rate for $5 \mathrm{~min}$. The needle was withdrawn 10 min later and the scalp sutured. Mice were monitored for 4-6 h after recovery from anesthesia and were perfused with $4 \%$ PFA $48 \mathrm{~h}$ after injection. The brains were removed and sectioned for immunohistochemistry.

Statistical analysis. All values are expressed as the mean of at least three determinations \pm SEM. Data were analyzed by Student's $t$ test and $p<$ 0.05 was considered to indicate statistical significance.

\section{Results}

\section{Astrogliosis is an early event in p25 transgenic mice}

To investigate the early changes in gene expression by p25 overexpression in vivo, we performed microarray analyses of 4 week induced p25Tg mice forebrain samples and found robust elevation of inflammatory markers such as GFAP, proinflammatory cytokines and chemokines. This suggested that neuroinflammation is an early phenomenon in p 25 overexpressed mice (data not shown). To confirm this, Western blot analyses (Fig. $1 A, B$ ) and immunohistochemistry (Fig. $1 C$ ) were performed on brain samples of $1,4,8$ and 12 week induced p25Tg/control mice (age matched noninduced p25Tg mice) using mouse anti-GFAP antibody. Robust increases in GFAP expression was observed in all the induction periods of p25Tg mice compared with their controls even at 1 week of induction of p25 expression. We also performed quantitative (q)RT-PCR studies for the inflammatory cytokines and chemokines such as MIP- $1 \alpha$, TNF- $\alpha$, TGF- $\beta$ and IL- $1 \beta$ in the brain samples of $1,4,8$ and 12 week induced p25Tg/ control mice and found significant increases in these cytokines and chemokines in all p25Tg mice samples compared with the controls (Fig. $1 D, E$ ). Collectively, our results suggest that activation of glia and subsequent chemokine production are very early events in p25Tg mice. To validate the involvement of Cdk5 in p25-mediated neuroinflammation, kinase assays were performed on p25Tg mice brain samples and the results confirmed the hyperactivation of Cdk5 in all the induction points of p25 expression (Fig. $1 F$ ). In addition, Cdk5 levels were found to be unchanged using Western blot analyses (Fig. 1G).

\section{p25-induced astrogliosis is an $\boldsymbol{\beta}$-amyloid independent event and occurs before microgloisis in $\mathrm{p} 25 \mathrm{Tg}$ mice}

Previous studies suggesting that amyloid plays a key role in the induction of neuroinflammation (Combs et al., 2001; White et al., 2005). To investigate the role of amyloid and phospho-tau in the initiation of p25-mediated neuroinflammation, we examined time points of $\beta$ amyloid production and phospho tau in $\mathrm{p} 25 \mathrm{Tg}$ mice. Hyperphosphorylation of tau and amyloid pathology were seen only in 4 week and 8 week induced p25Tg mice respectively (Fig. 2A). However, astrogliosis and chemokine production were found even in 1 week of p25 induction (Fig. 1). Our results indicate that astrogliosis induced by p 25 overexpression might be independent of amyloid pathology. To study the microglial activation status in $\mathrm{p} 25 \mathrm{Tg}$ mice, we performed immunohistochemistry with the brain sections of 1, 4, 8 and 12 week induced p25Tg/ control mice using anti-Cd11b antibody (a marker for activated microglia) (Matsuoka et al., 2001) (Fig. 2B). Previous studies reported that microglial activation can be induced by neuronal tissue plasminogen activator (tPA), a serine protease that catalyzes the conversion of inactive plasminogen to the active protease plasmin (Takahashi et al., 2010). To study the role of tPA in the microglial activation, we have also performed the immunohistochemistry with tPA antibody. These results mirror the Cd11b findings (Fig. $2 \mathrm{~B}$ ). Together, our results show that microgloisis was absent at 1 week of p25 induction in p25Tg mice and only became apparent at later time points. To further investigate the involvement of peripheral cells in p25-induced neuroinflammation, immunohistochemistry was performed in p25Tg/control mice brain sections with CD4 and CD8 antibodies and we found dramatic increases in these markers in p25Tg mice compared with control mice (Fig. 2C). Our results suggest that there is peripheral cell infiltration during p25-mediated neuroinflammation.

\section{p25-induced astrocyte activation is mediated by a soluble factor}

To explore how p25 overexpression mediates neuroinflammation and the nature of the factor that is responsible for astrogliosis, glia were cocultured with neurons transduced with p25-LV/empty vector (EV-LV) and primary neurons from p25Tg/control mice. Significant increases in GFAP staining 
A

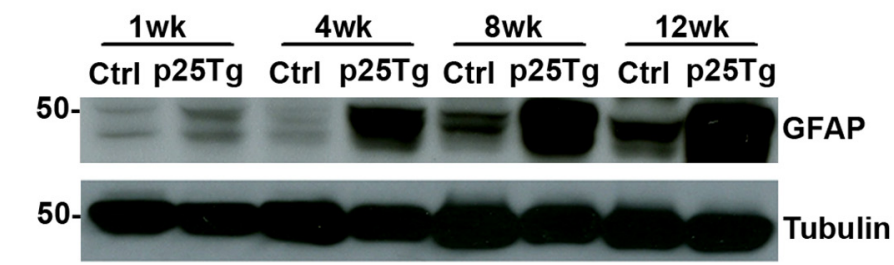

C

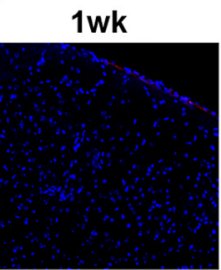

4wk

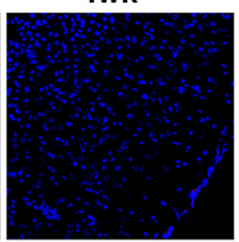

8wk
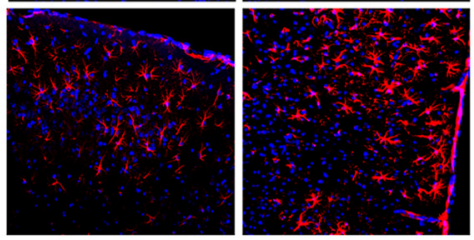

$\mathbf{E}$

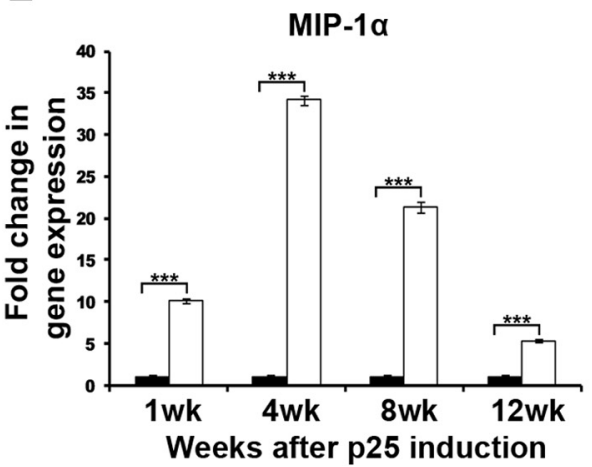

F
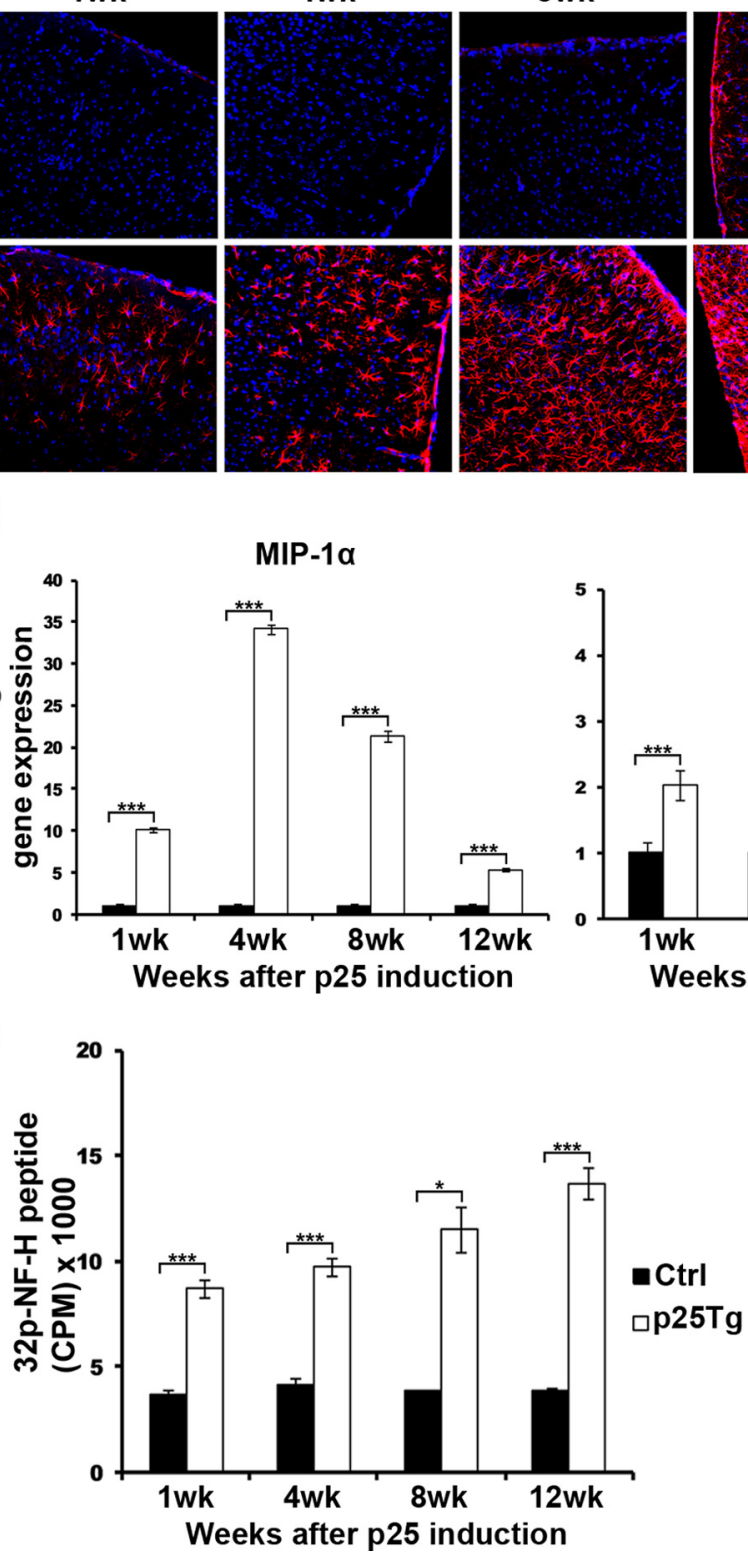

G
B

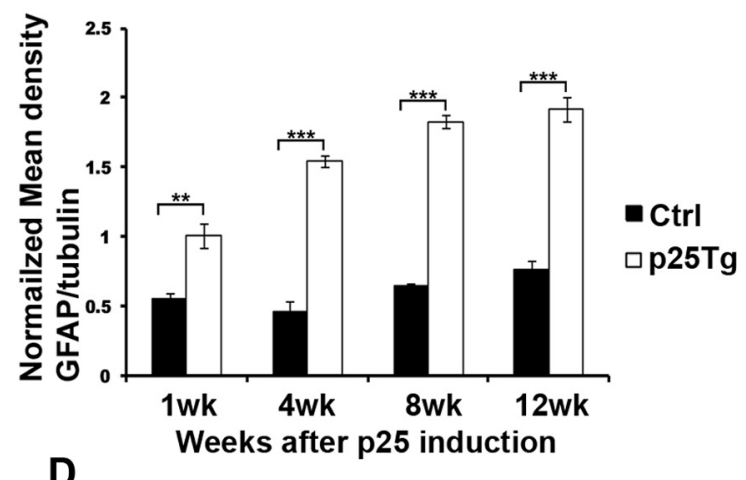

D
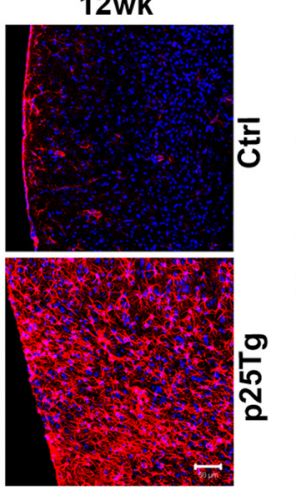

TNF- $\alpha$

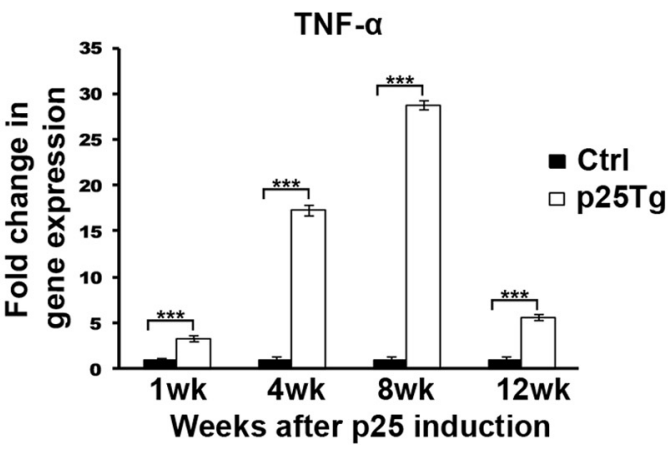

IL-1 $\beta$
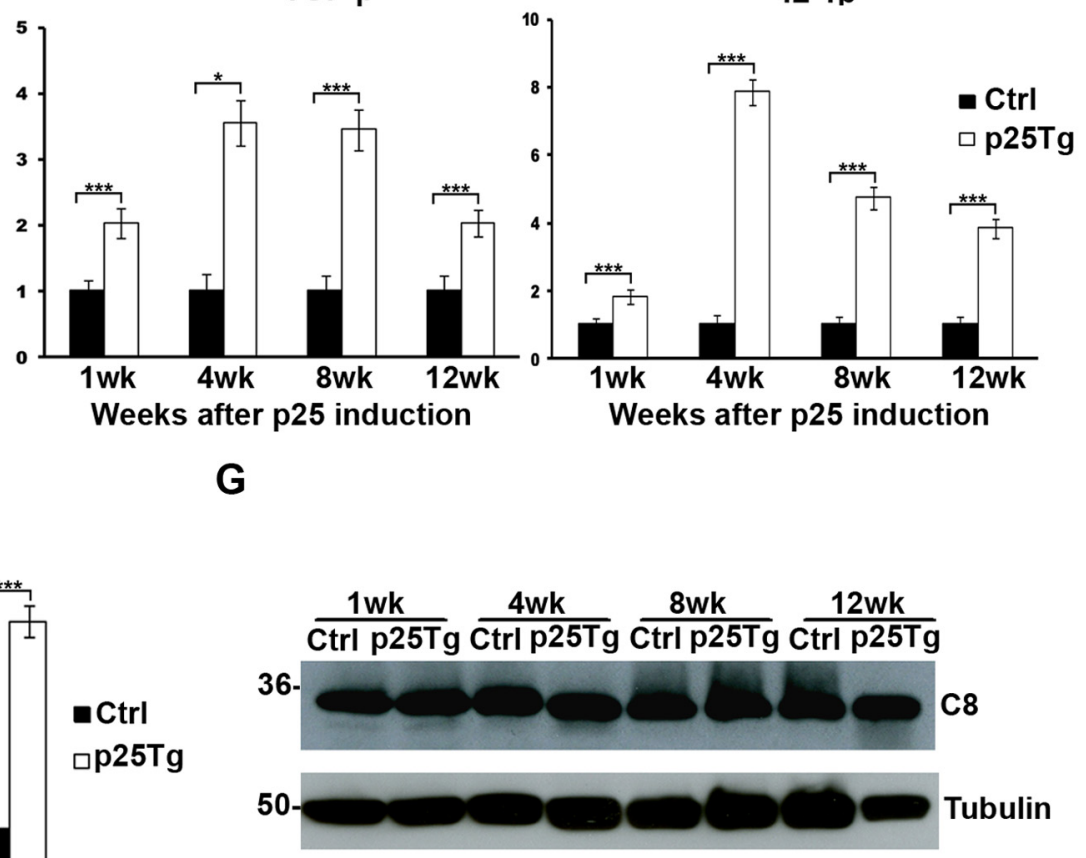

Figure 1. Neuroinflammation is an early event in p25 transgenic mice. $\boldsymbol{A}$, Immunoblot analyses were performed on the brain lysates of 1, 4, 8 and 12 week induced p25 transgenic mice (p25Tg) and their respective age-matched noninduced p25Tg control mice (Ctrl) using anti-GFAP antibody (top). Equal amounts of protein loading were confirmed by reprobing the membrane with anti-tubulin antibody (bottom). B, Quantification of immunoblot analyses in $A$ by densitometric scanning ( ${ }^{* *} p<0.01$ and ${ }^{* * *} p<0.001$ ). C, Representative confocal images of the cortex from $1,4,8$ and 12 week induced $p 25$ Tg and control mice brains. The sections were immunostained with anti-GFAP antibody (red) and DAPI (blue). Scale bars represent $50 \mu$ m and images are representative of $n=3$ mice. $\boldsymbol{D}, \boldsymbol{E}$, Real-time PCR (RT-PCR)

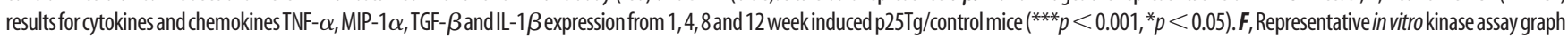
using active kinase (Cdk5) from p25Tg/control mice brain lysates to phosphorylate a NF-H peptide ( ${ }^{* * *} p<0.001,{ }^{*} p<0.05$ ). G, Western blot analyses of the brain lysates from p25Tg/control mice using anti-Cdk5 (C8) antibody. Error bars indicate +SEM. 
A
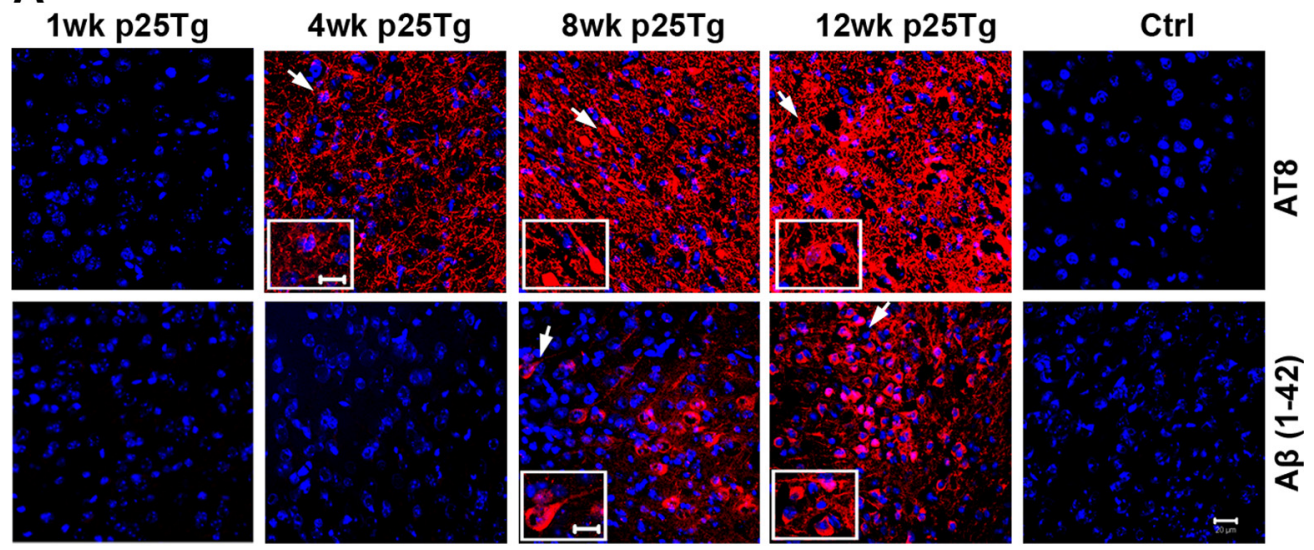

\section{B 1wk p25Tg}
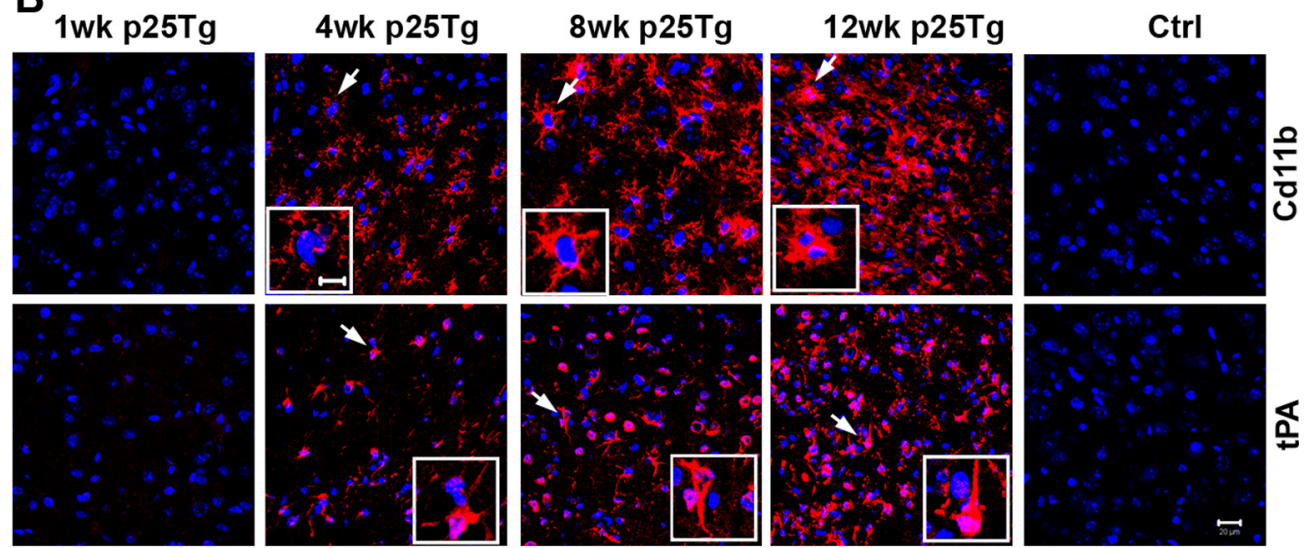

C
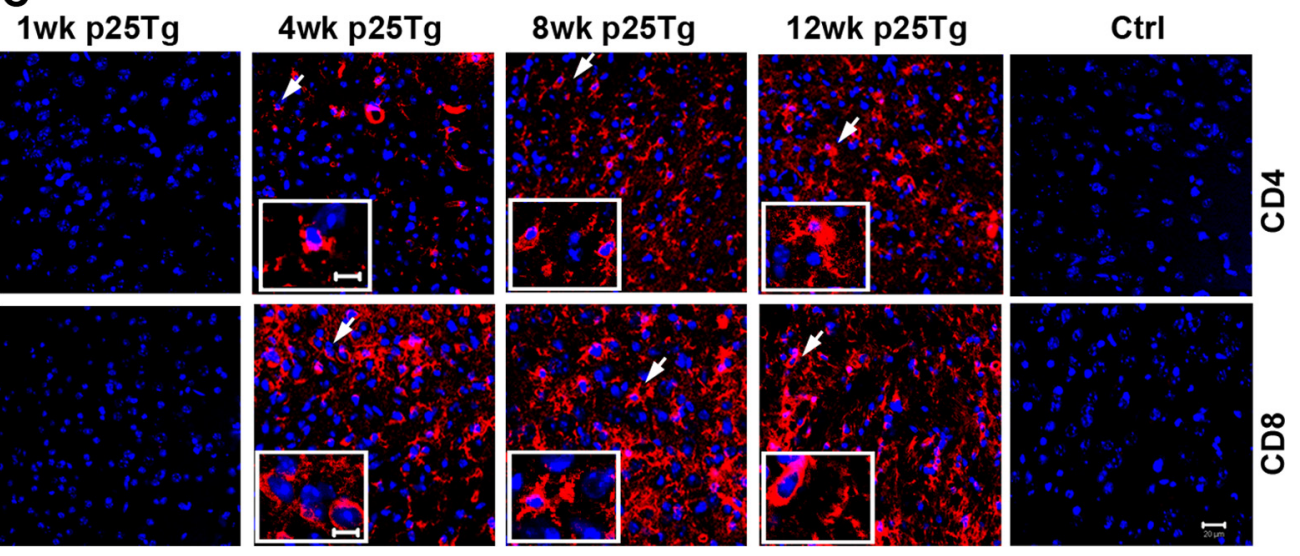

Figure 2. Initiation of neuroinflammation is independent of $\beta$ amyloid and tau phosphorylation in $p 25$ transgenic mice. $A-C$, Representative confocal images of the cortex from $1,4,8$ and 12 week induced p25Tg and control mice (age matched for 12 week time point). The brain sections were immunostained with (A) phospho-tau (AT8) and A $\beta$ 1-42, (B) anti-Cd11b and anti-tPA, and (C) CD4 and CD8 (red) antibodies. The nuclei were counterstained with DAPI (blue). White arrows indicate the region that is enlarged in high-magnification insets. Scale bars: (main panel), $20 \mu \mathrm{m}$; (insets), $10 \mu \mathrm{m}$. Images are representative of $n=3$ mice.

was seen in glia that were cocultured with neurons transduced with $\mathrm{p} 25-\mathrm{LV}$ and neurons from $\mathrm{p} 25 \mathrm{Tg}$ mice compared with the respective controls. Our immunocytochemistry results showed that the factor produced by $\mathrm{p} 25$ overexpression was soluble since the neurons and glia were not in contact with each other (Fig. 3A). These results were confirmed by Western blots of glial samples that were cocultured with neurons transduced with EV-LV/p25-LV or neurons from p25Tg/control mice (Fig. $3 B$ ) where the outcome mirrored the immunostaining results described above. We also performed qRT-PCR on glia that were cocultured with neurons transduced with EV-
$\mathrm{LV} / \mathrm{p} 25-\mathrm{LV}$ and the neurons from $\mathrm{p} 25 \mathrm{Tg} / \mathrm{control}$ mice for the inflammatory cytokines and chemokines such as MIP- $1 \alpha$, TNF- $\alpha$, TGF- $\beta$ and IL- $1 \beta$. Significant increases in cytokine and chemokine levels were seen in glia that were cocultured with p25 overexpressing neurons compared with the controls (Fig. $3 C, D$ ). Hyperactivation of Cdk5 due to $\mathrm{p} 25$ overexpression in $\mathrm{p} 25$-LV-transduced cortical neurons was validated using kinase assays and Western blot analyses (Fig. 3E,F). To further confirm the soluble nature of the factor causing reactive gliosis, we transferred cell-free supernatants from neurons transduced with EV-LV/p25-LV and neurons from 
A

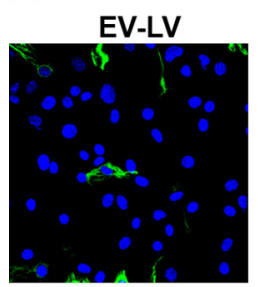

Ctrl

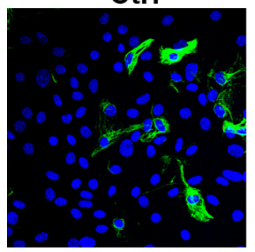

D

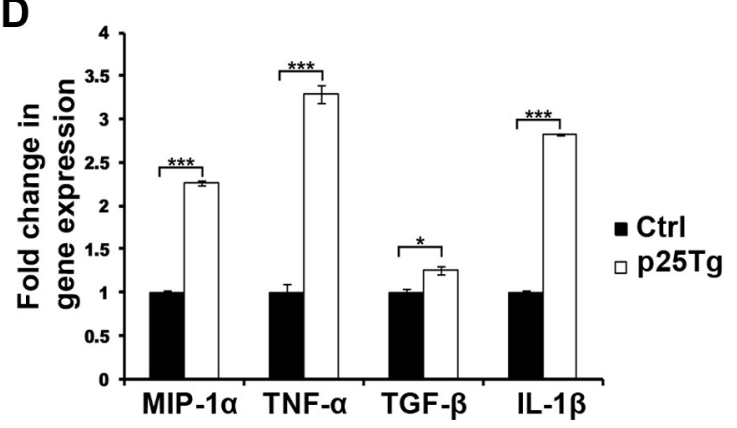

G
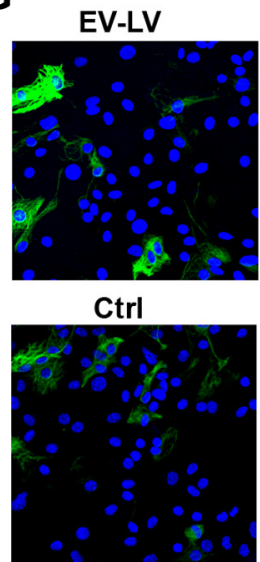

B

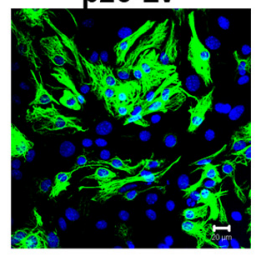

p25Tg

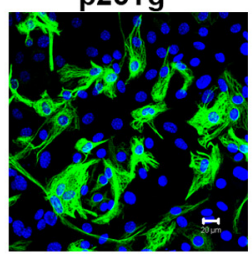

p25Tg

C
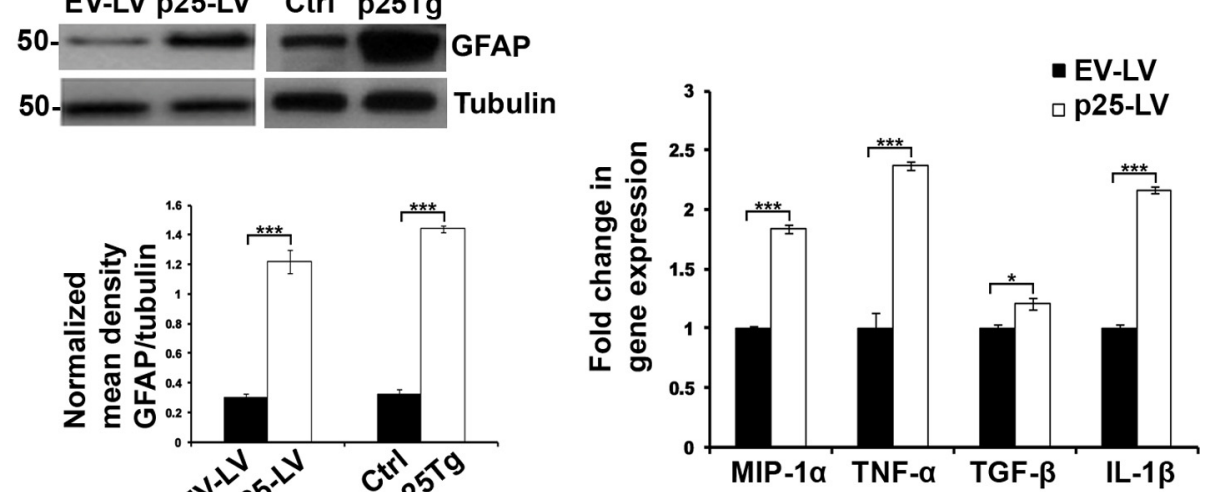

E

F
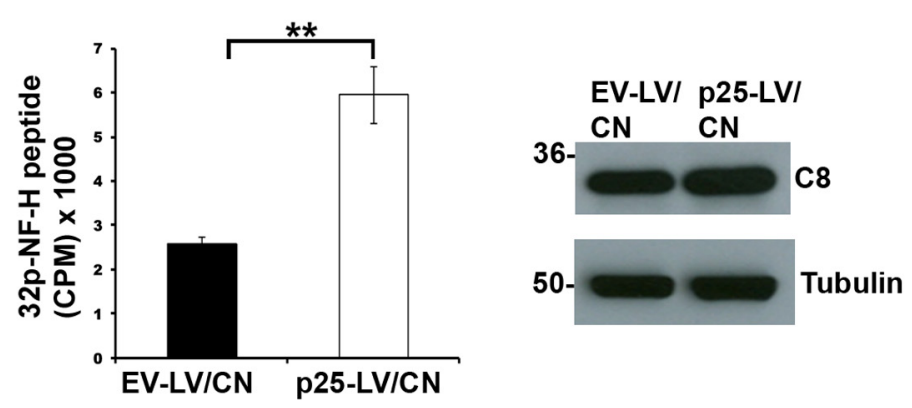

H

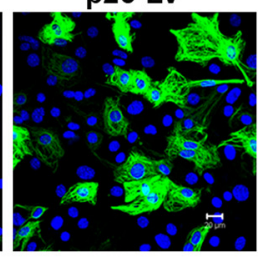

EV-LV p25-LV Ctrl p25Tg

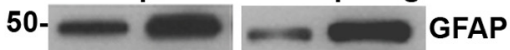

50-
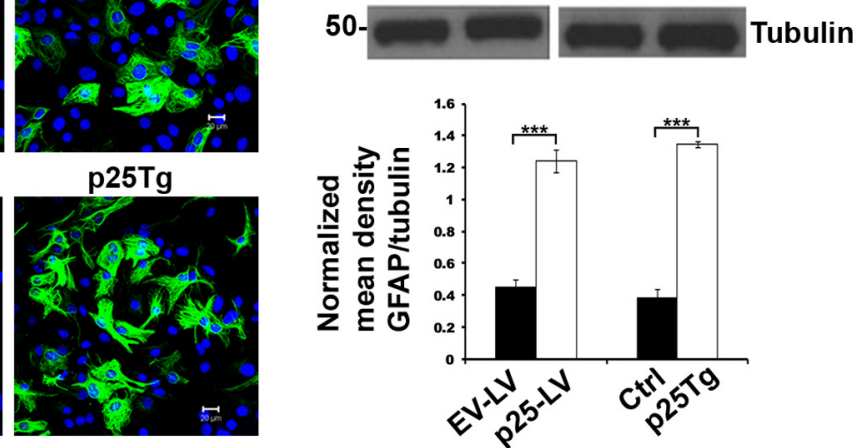

Figure 3. A soluble factor mediates p25-induced neuroinflammation. A, 7-DIC cortical neurons transduced with EV-LV/p25-LV and primary neurons from control/5 dinduced p25Tg mice were cocultured with glia for $48 \mathrm{~h}$ using tissue culture inserts. Glia were fixed and immunostained with anti-GFAP antibody (green) and DAPI (blue). Scale bars, $20 \mu \mathrm{m}$. B, Immunoblot analyses of lysates from glia cocultured with EV-LV/p25-LV-transduced neurons and neurons from control/p25Tg mice. The samples were immunoprobed with anti-GFAP antibody (top). Tubulin serves as a loading control (bottom). Bar graphs show the quantification of GFAP levels (*** $p<0.001)$. C, D, RT-PCR results showing the expression of cytokines and chemokines MIP-1 $\alpha$, TNF- $\alpha$, TGF- $\beta$, and IL- $1 \beta$ in glia cocultured with cortical neurons transduced with EV-LV/p25-LV and cortical neurons from control/ p25Tg mice ${ }^{* * *} p<0.001$ and $\left.{ }^{*} p<0.05\right)$. $E$, Representative in vitro kinase assay graph using active kinase (Cdk5) from cortical neurons transduced with EV-LV/p25-LV to phosphorylate a NF-H peptide $\left({ }^{* *} p<0.01\right)$. $F$, Western blot analyses of the lysates from cortical neurons transduced with EV-LV/p25-LV using anti-Cdk5 (C8) antibody. G, Cell-free supernatants from EV-LV/p25-LV-transduced neurons and neurons from control/p25Tg mice were transferred to glia for $48 \mathrm{~h}$. The glia were fixed and immunostained with antibody to GFAP (green) and DAPI (blue). Scale bars represent $20 \mu \mathrm{m}$. $\boldsymbol{H}$, Western blot analyses of lysates of glia incubated with cell-free supernatants from neurons transduced with EV-LV/p25-LV and control/p25Tg mice neurons using antibody to GFAP. Bar graph shows the quantification of GFAP levels $\left({ }^{* * *} p<0.001\right)$. Error bars indicate \pm SEM.

p25Tg/control mice to glia. The immunostaining and Western blot analyses results supported our finding that a soluble signal from p25 overexpressed neuron causes glial activation (Fig. 3G,H).

p25 overexpressed neurons activate glia through a lipid signal To characterize the biochemical nature of the secreted factor from the p25 overexpressed neurons, cell-free supernatants of neurons transduced with EV-LV/p25-LV were treated with DNase, RNase and Proteinase K to systematically remove possible factors that could be secreted into solution during inflammation (Lauber et al., 2003). These enzyme-treated supernatants were then transferred to glia and incubated for $48 \mathrm{~h}$ and glia were processed for immunostaining (Fig. $4 A, B$ ) and Western blot analyses (Fig. 4C,D). The increase in GFAP levels in glia that received DNase-, RNase-, and Proteinase K-treated supernatants 
A
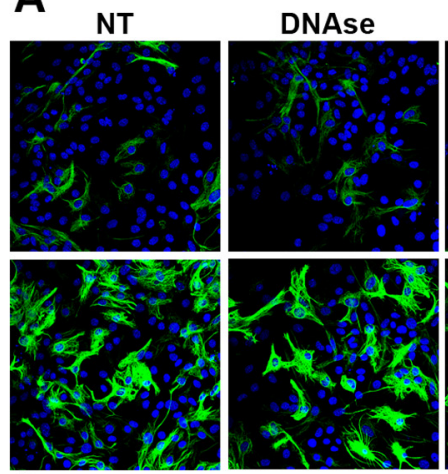

C

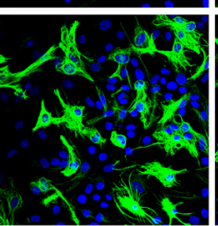

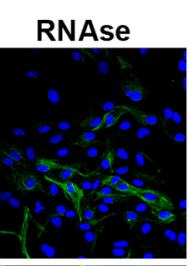

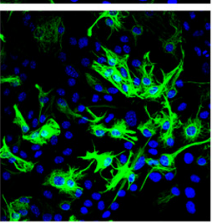

Proteinase $\mathrm{K}$
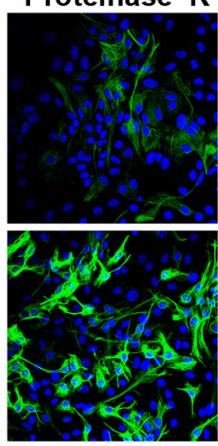

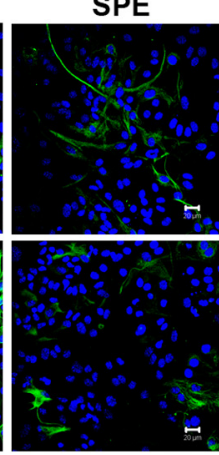

B

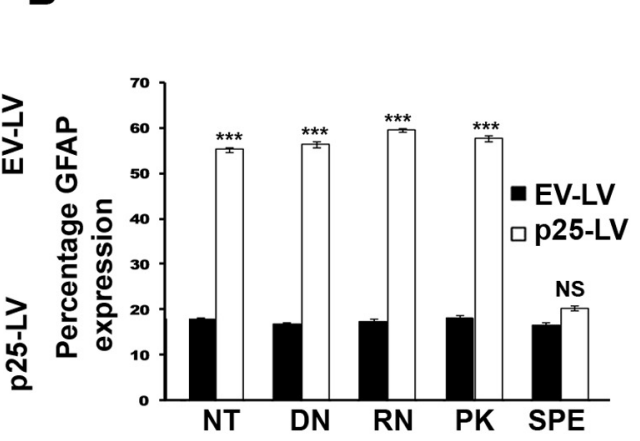

D

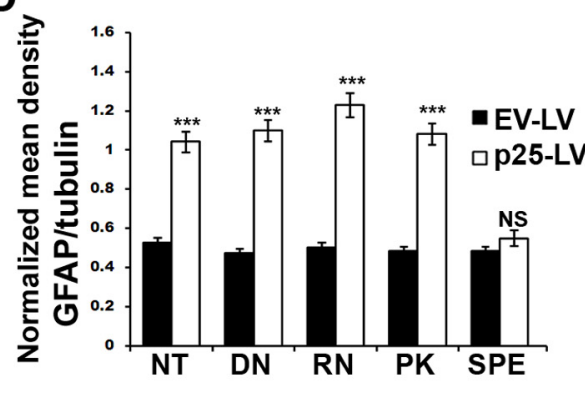

E

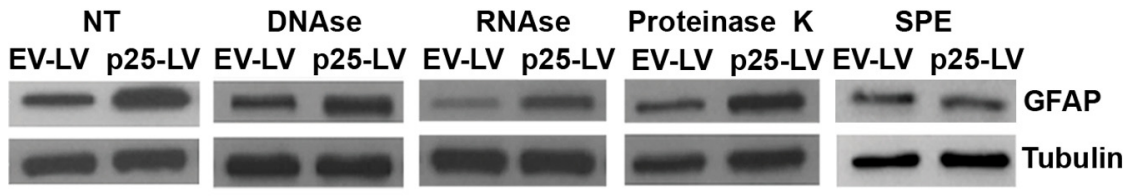
MIP-1a TNF- $\alpha$ TGF- $\beta$

IL-1 $\beta$

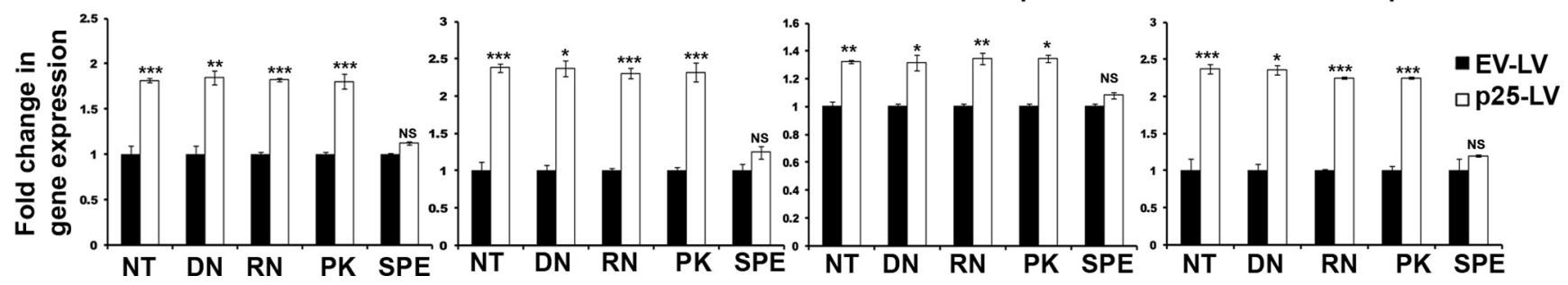

Figure 4. p25-induced neuroinflammation is mediated by a soluble lipid. $A$, Cell-free supernatants from EV-LV/p25-LV-transduced neurons treated with DNase (DN), RNase (RN) and Proteinase K (PK) for 60 min or passed through SPE-C18 columns to remove lipids, were transferred to glia for $48 \mathrm{~h}$. The nontreated (NT) and treated glia were fixed and immunostained with anti-GFAP antibody (green) and DAPI (blue). Scale bars, $20 \mu \mathrm{m}$. B, Quantification of GFAP expression in $\boldsymbol{A}$ ( ${ }^{* * *} p<0.001$; NS, $p>0.05$ ). $\boldsymbol{C}$, Lysates of glia that received same treatment as in $\boldsymbol{A}$ were separated by SDS-PAGE and immunoprobed with anti-GFAP antibody. Tubulin acts as a loading control. $\boldsymbol{D}$, Quantification of immunoblot analyses in $\boldsymbol{C}\left({ }^{* * *} p<0.001\right.$, NS, $\left.p>0.05\right)$. $\boldsymbol{E}$, RT-PCR results showing the expression of cytokines and chemokines MIP- $1 \alpha$, TNF- $\alpha$, TGF- $\beta$, and IL- $1 \beta$ in glia that received the same treatment as in $\boldsymbol{A}\left({ }^{* * *} p<0.001\right.$, ${ }^{* *} p<0.01,{ }^{*} p<0.05$, NS, $\left.p>0.05\right)$. Error bars indicate \pm SEM.

from the p25-overexpressed neurons showed that neither DNA, RNA, nor protein was likely to be responsible for p25mediated neuroinflammation. To further elucidate the nature of the factor, we removed major species of lipid from the supernatants via SPE-C18 column elution and transferred these to glia. The significant reduction in GFAP levels in glia that received the column eluted supernatants suggested that the soluble factor from the p25 overexpressed cells could be lipid in nature (Fig. $4 A-D$ ). qRT-PCR analyses for chemokines on the glia that received DNase-, RNase-, and Proteinase-treated supernatants or SPE-C18 column eluted supernatants from p25 overexpressing neurons further confirmed our findings and dramatic reductions in chemokines were found in SPE-C18-treated supernatants (Fig. 4E).

LPC mediates the p25/Cdk5-induced inflammatory cascade To further confirm the lipid nature of the factor responsible for p25-induced astrogliosis, we extracted lipids from the supernatants of neurons transduced with EV-LV/p25-LV and transferred the lipids onto glia for 24 and $48 \mathrm{~h}$. Immunocytochemical staining (Fig. 5A) and Western blot analyses (Fig. 5B,C) using antiGFAP antibody showed significant increases in GFAP expression in the glia treated with lipids from p25-transduced neurons. To validate our finding in vivo, we stereotactically injected the lipids from p25-transduced neurons into mice brain and found a robust increase in GFAP immunoreactivity compared with mice injected with lipids from empty vector-transduced neurons (Fig. $5 D)$. To further determine the identity of the lipid found in the supernatants of the p25 overexpressed neurons that caused this astrogliosis, lipidomic mass spectrometric analysis was performed on the lipids extracted from the supernatants of neurons transduced with EV-LV/p25-LV. Among the major lipids, LPC levels showed a significant increase in lipids derived from p25LV-transduced neurons compared with the EV-LV-transduced neurons. Although LysoPI levels were also elevated, the difference was not statistically significant $(p=0.223$ ) (Fig. $5 E$ ). Lipidomic mass spectrometry using internal standards for various LPC isoforms further identified LPC 16:0, 18:0 and 18:1 as the major lipids elevated in p25-LV derived lipids compared with controls (Fig. 5F). To determine which LPC isoform is more potent in the induction of GFAP expression, we treated glia with commercial LPC 16:0, 18:0 and 18:1. Our results show that LPC 18:1 was the most potent lipid to cause GFAP upregulation even at $24 \mathrm{~h}$ (Fig. 5G-I). The amount of LPC in the cell-free supernatants of p25 overexpressed cells were $\sim 10$-fold lower than the 
A

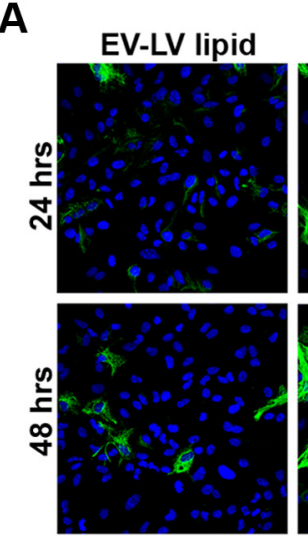

D
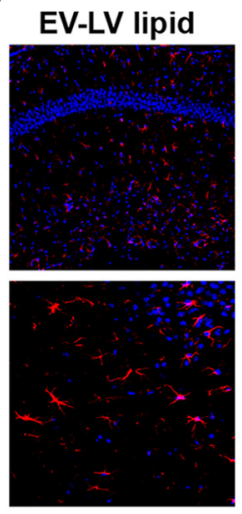

$\mathbf{F}$

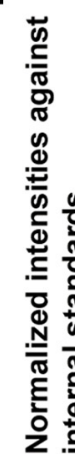

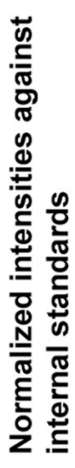

B
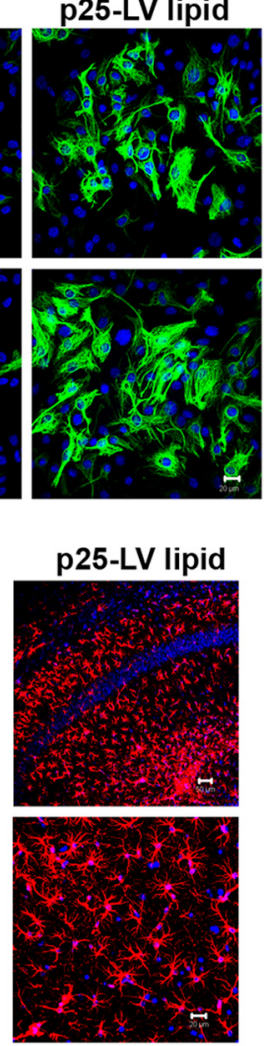

E

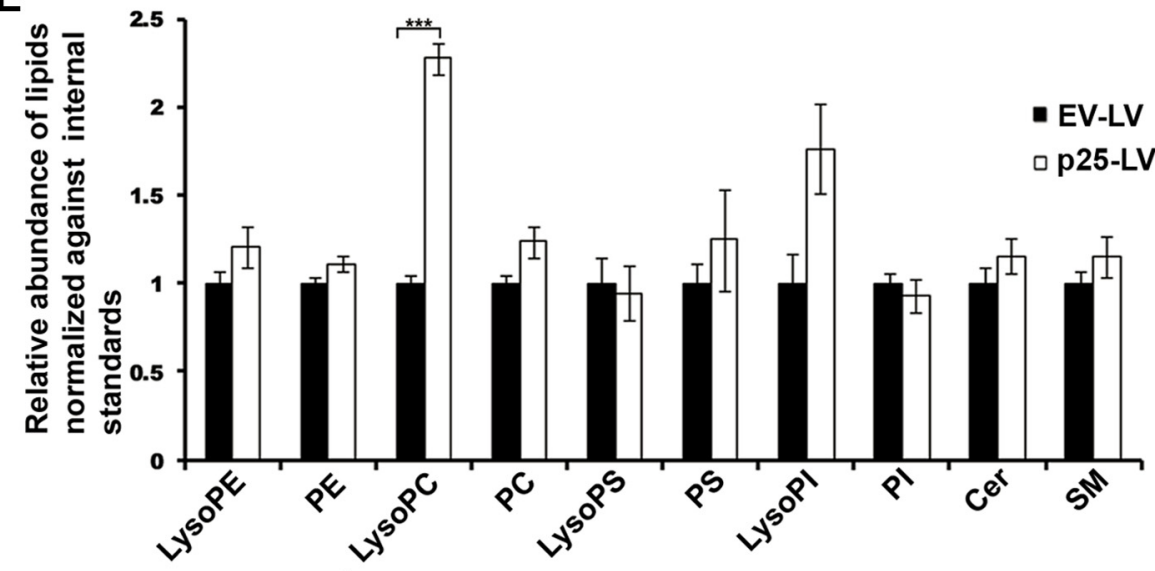

EV-LV lipid p25-LV lipid $24 \mathrm{hrs} 48 \mathrm{hrs} 24 \mathrm{hrs} 48 \mathrm{hrs}$

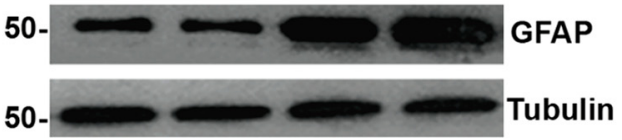

G
C

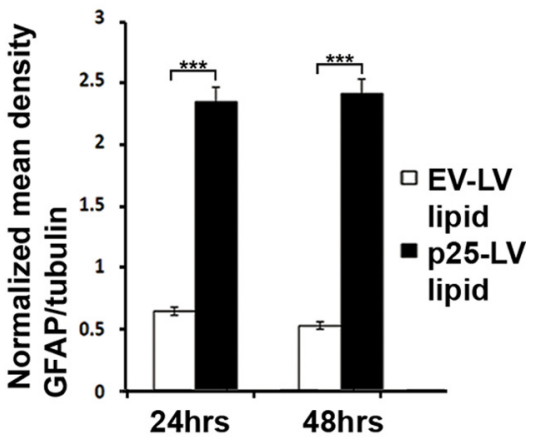

$\mathbf{H}$
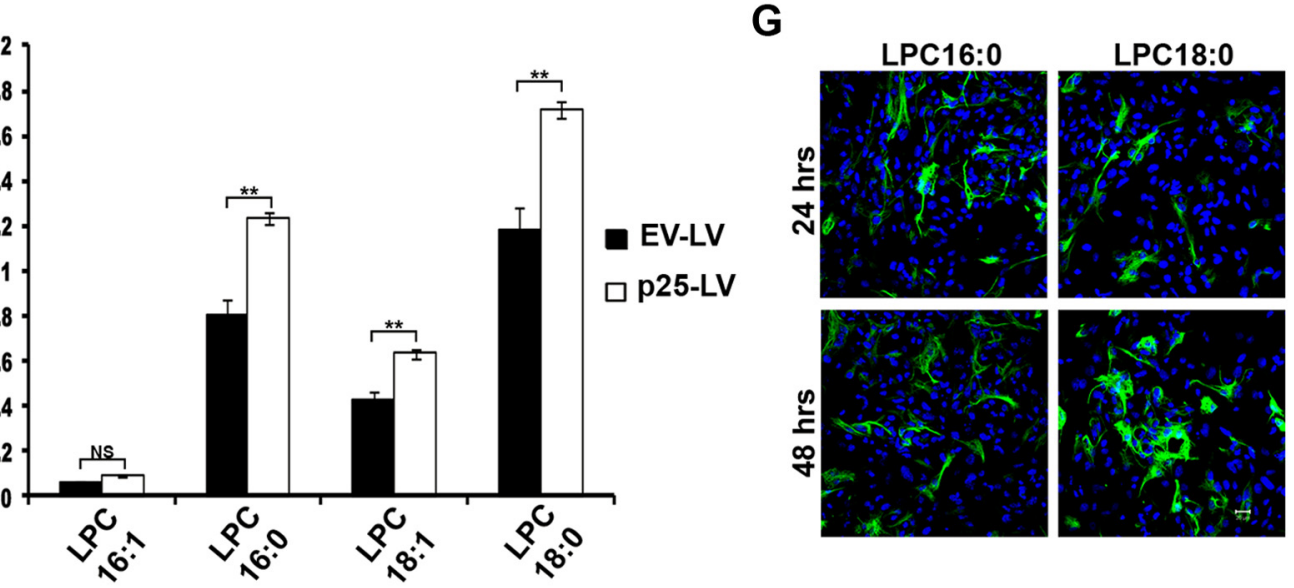

LPC18:1
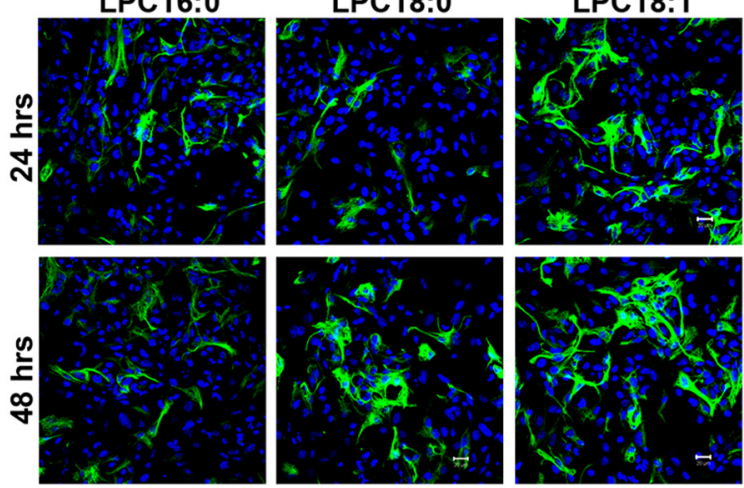

I
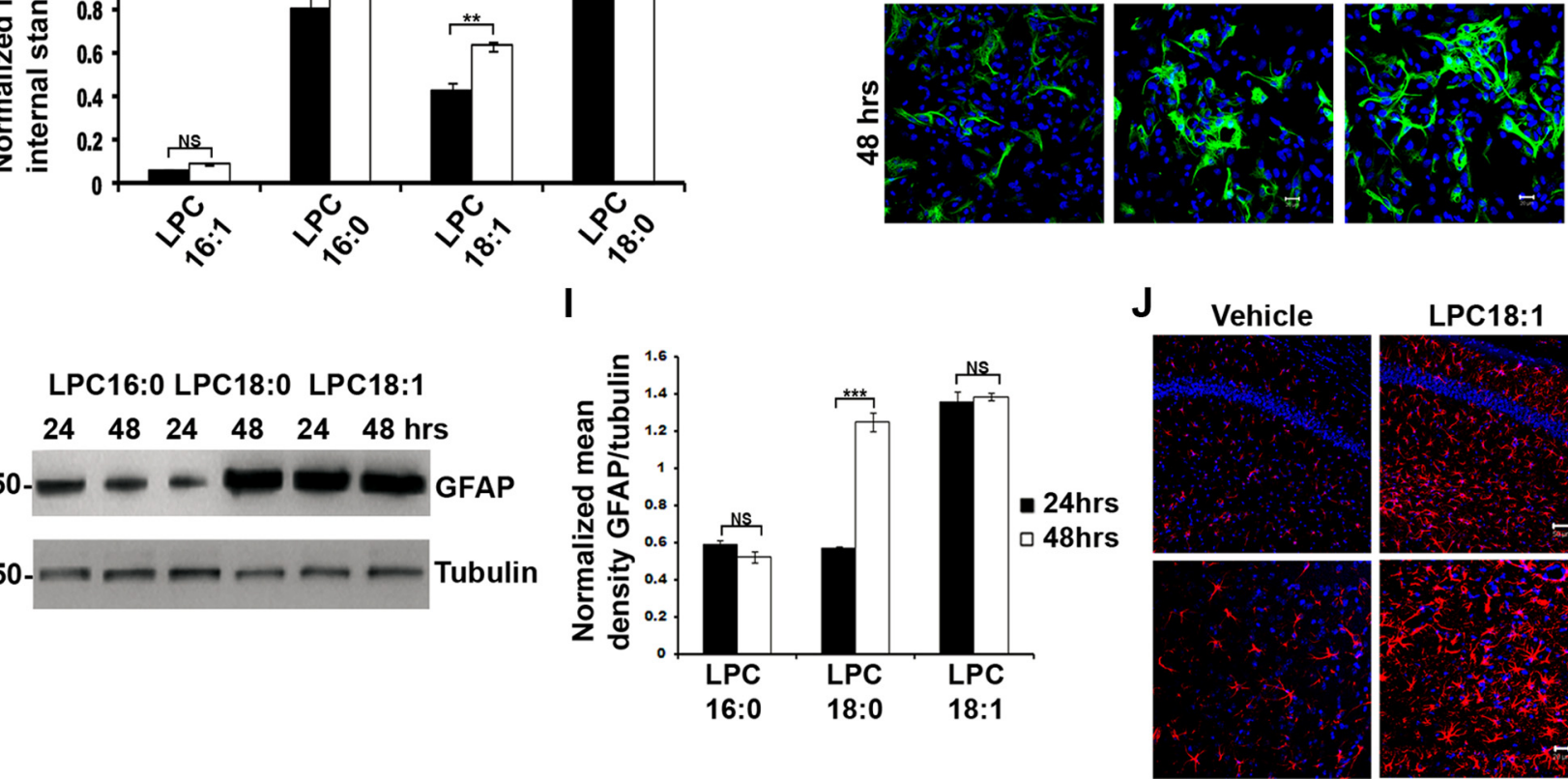

LPC18:1

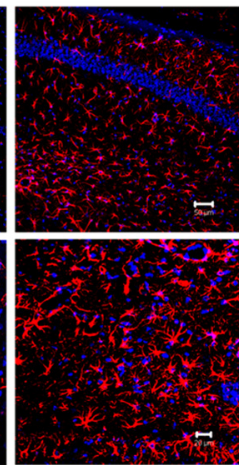

Figure 5. p25 overexpressing cells secrete LPC that mediates astrogliosis. $A$, Glia incubated with lipids extracted from supernatants of neurons transduced with EV-LV/p25-LV for 24 and $48 \mathrm{~h}$ were fixed and immunostained with anti-GFAP antibody (green) and DAPI (blue). Scale bars, $20 \mu \mathrm{m}$. $\boldsymbol{B}$, Immunoblot analyses of lysates from the glia samples that received identical treatment as in $\boldsymbol{A}$ using anti-GFAP antibody. C, Quantification of immunoblot analyses in $\boldsymbol{B}\left({ }^{* *} p<0.001\right)$. $\boldsymbol{D}$, Lipids from supernatants of neurons transduced with EV-LV/p25-LV (Figure legend continues.) 
amount of commercial LPC required to activate the glial cells. The form of LPC in the supernatant might be more potent and form an active micelle more readily than the commercially available LPC. Additive effect of other lipids in the supernatant of p25 overexpressed neurons may also be responsible for this increase potency. It would be hard to determine this additive effect due to the combination of various other lipids where the permutations are numerous. Our finding was supported by a previous study describing 100-fold differences between the amount of LPC in supernatants and commercial LPC required for chemotactic activity (Lauber et al., 2003). To investigate the in vivo relevance of LPC in astrogliosis, stereotactic injections of commercial LPC 18:1 and vehicle were performed in mouse brains (Fig. 5J) and the results mirror the in vitro experiment findings. Although some studies used PBS to dissolve commercial LPC (Sheikh et al., 2009), Chloroform/Methanol (1:2) was used as a vehicle in our experiments to keep the solvent constant for the commercial LPC as well as the lipid extract treatments. Lipid extract did not dissolve in PBS due to the poor hydrating nature of the lipids other than LPC. Lipidomic mass spectrometric analysis on p25Tg mice brain samples further validated our findings where LPC levels were significantly elevated compared with controls (Fig. 6A). Collectively, our in vivo and in vitro results revealed that LPC is one of the major lipid secreted by the p 25 expressing neurons to result in astrogliosis.

\section{p25-induced upregulation of cPLA2 causes LPC production}

We investigated the changes in CPLA2 expression and activity in p25 overexpressed neurons to determine the mechanism behind p25-mediated LPC production. RT-PCR results showed robust increases in cPLA2 expression in different induction periods of p25Tg mice brain samples (Fig. 6B). We also found significant increases in cPLA2 expression as well as cPLA2 activities in p25$\mathrm{LV}$-transduced neurons and $5 \mathrm{~d}$ induced $\mathrm{p} 25 \mathrm{Tg}$ mice neurons (Fig. 6C-F). The involvement of other PLA2 isoforms in p25induced LPC production was investigated using RT-PCR studies for iPLA2 expression, inhibitor studies using BEL (iPLA2 inhibitor) and AACOCF3 (cPLA2 inhibitor). In these experiments, cPLA2 was found to be the principle enzyme involved in p25mediated LPC production (Fig. 6G,H). To further confirm the involvement of p25-induced upregulation of cPLA2 in LPC production, cPLA2 gene silencing experiments using cPLA2 shRNA lentiviral particles in $\mathrm{p} 25$-LV-transduced neurons were performed. Silencing was validated by qRT-PCR, cPLA2 activity assays and Western blots (Fig. 6I,J).

\footnotetext{
(Figure legend continued.) were stereotactically injected into mice brains and the brain sections were immunostained with anti-GFAP antibody (red) and DAPI (blue). Scale bars: (20X, top), $50 \mu \mathrm{m} ; 20 \mu \mathrm{m}$ (40X, bottom), $20 \mu \mathrm{m}$. $\boldsymbol{E}$, Mass spectrometric analyses were performed on lipids from supernatants of neurons transduced with EV-LV/p25-LV. Results were normalized against the internal standards of the respective lipids $\left({ }^{* * *} p<0.001\right)$. $\boldsymbol{F}$, Mass spectrometric analyses were performed on lipids from samples as in $\boldsymbol{E}$ and the results were normalized against the internal standards of the respective LPC isoforms $\left({ }^{* *} p<0.01 ;\right.$ NS,$\left.p>0.05\right)$. G, Glia were treated with media containing $20 \mu \mathrm{M} \mathrm{LPC}$ of 16:0, 18:0, or 18:1 for 24 and $48 \mathrm{~h}$. Immunocytochemistry of glia was performed using anti-GFAP antibody (green) and DAPI (blue). Scale bars, $20 \mu \mathrm{m}$. $\boldsymbol{H}$, Western blot analyses of lysates from the samples as in $\boldsymbol{G}$ using anti-GFAP antibody. I, Quantification of immunoblot analyses in $\boldsymbol{H}{ }^{* * *} p<0.001$; NS, $\left.p>0.05\right)$. J, LPC 18:1/vehicle was injected into mice brains and immunostaining was performed using antiGFAP antibody (red) and DAPI (blue). Scale bars: (20X, top), $50 \mu \mathrm{m}$; (40X, bottom), $20 \mu \mathrm{m}$. Error bars indicate \pm SEM.
}

\section{cPLA2 knock-down attenuates the p25-mediated glial} activation and chemokine production

To investigate the effect of cPLA2 knock-down in p25-mediated LPC production, mass spectrometric analyses were performed and results showed a significant decrease in LPC 18:0 and 18:1 levels in lipid extracts from the $\mathrm{p} 25-\mathrm{LV}+\mathrm{cPLA} 2$ shRNA-transduced neurons compared with controls (Fig. 7A). To further study the effect of cPLA2 silencing in p25-mediated neuroinflammation, supernatants from p25-LV+ cPLA2 shRNA/p25-LV+ control shRNAtransduced neurons were transferred to glia. Western blot analyses and immunostaining were performed using anti-GFAP antibody after $48 \mathrm{~h}$ of incubation. Marked reductions in GFAP staining were observed in glia that were incubated with the supernatants from p25-LV+cPLA2 shRNA-transduced neurons compared with controls (Fig. 7B-D). We also performed qRT-PCR with the glia treated with cell-free supernatants from p25-LV + cPLA2 shRNA or p25$\mathrm{LV}+$ control shRNA-transduced neurons for MIP- $1 \alpha$, TNF- $\alpha$, TGF- $\beta$ and IL- $1 \beta$. Decreases in chemokine and cytokine expression levels in glial samples that received supernatants from p25LV + cPLA2 shRNA-transduced neurons compared with controls (Fig. 7E). To further confirm the effect of cPLA2 upregulation in the induction of neuroinflammation, glia were incubated with lipids extracted from supernatants of p25-LV+ control shRNA or p25$\mathrm{LV}+\mathrm{cPLA} 2$ shRNA-transduced neurons for $48 \mathrm{~h}$. Immunostaining and Western blot analyses results showed 4 to fivefold decreases in GFAP expression in glia treated with lipids from p25-LV+cPLA2 shRNA-transduced neurons compared with controls (Fig. $7 F-H$ ). We also injected lipids from the supernatants of p25-LV+ control shRNA and p25-LV+cPLA2 shRNA-transduced neurons into mice brains and immunostaining results showed robust reductions in GFAP expression in mice injected with lipids from $\mathrm{p} 25-\mathrm{LV}+\mathrm{cPLA} 2$ shRNA-transduced neurons (Fig. $7 I)$. Together, our results show that cPLA2 upregulation is a critical event in p25-mediated neuroinflammation.

\section{p25-induced inflammatory mediators trigger neurodegeneration}

To investigate the importance of p25-mediated neuroinflammation in the induction of neurodegeneration, we treated cortical neurons with conditioned media from glia that received lipids from EV-LV, p25-LV+ control shRNA and p25-LV+cPLA2 shRNA-transduced neurons. Robust increases in neurodegenerative markers such as phospho-tau (AT8) and intra cellular A $\beta 1-42$ accumulation in neurons that received supernatants from glia treated with lipids from p25 overexpressed neurons compared with glia treated with lipids purified from cPLA2 silenced p25 overexpressing neurons were seen (Fig. $8 A$ ). Results from in vivo injections of lipids from EV-LV, p25$\mathrm{LV}+$ control shRNA and p25-LV+cPLA2 shRNA-transduced neurons into mice brains show identical findings (Fig. $8 \mathrm{~B}$ ). We found significant increases in cell death in neurons that were treated with supernatants from glia incubated with lipids from p 25 overexpressed neurons (Fig. $8 C, D$ ). In contrast, we did not observe any cell death in our in vivo experiments (data not shown). This could be because of a single dose of lipids injected into mice and subsequent clearance of lipids as well as the abnormal protein species (tau and amyloid). In general, the crucial mechanism behind the neuronal death is the reduced clearance capability in neurons, probably attributed to the reduction in function of the proteosomal and autophagic machinery (Keller et al., 2000; Komatsu et al., 2006). Additionally, this trigger has to be sustained either by $\mathrm{p} 25$ production or by other toxic insults to cause neuronal damage. 
A

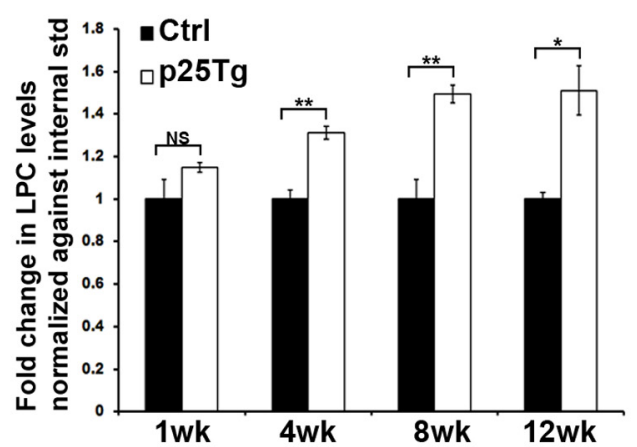

D

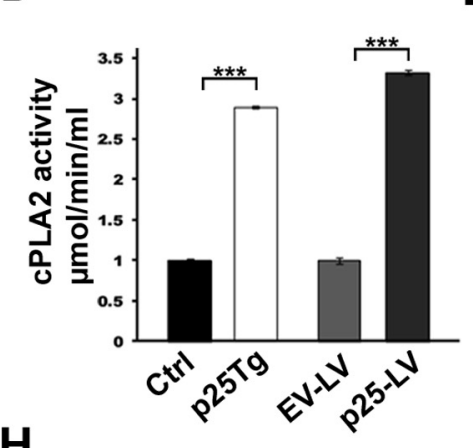

E

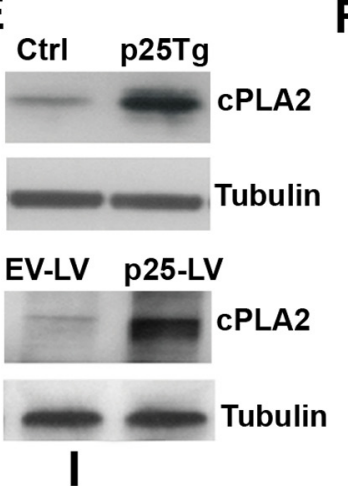

F

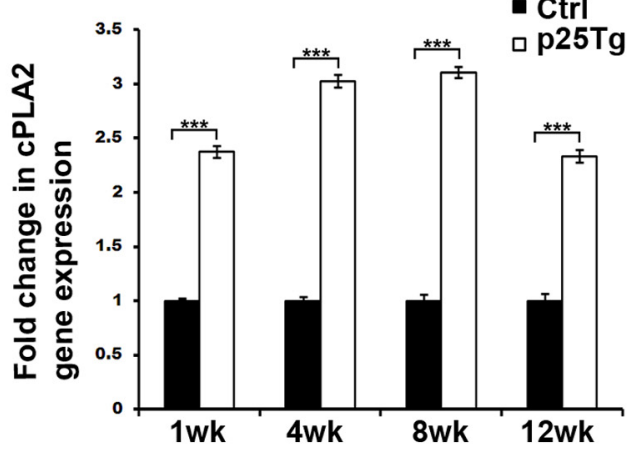

C
H

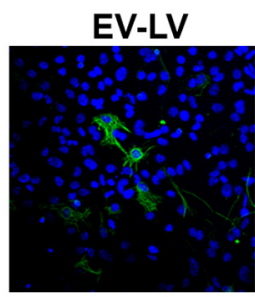

p25-LV+ AACOCF3

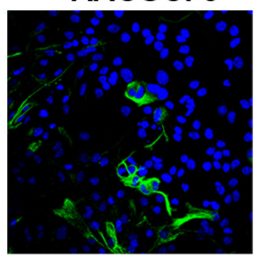

p25-LV

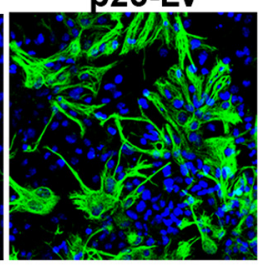

p25-LV+

BEL

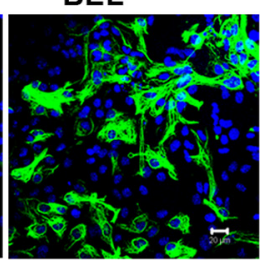

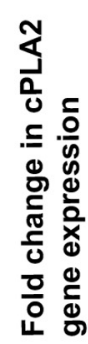

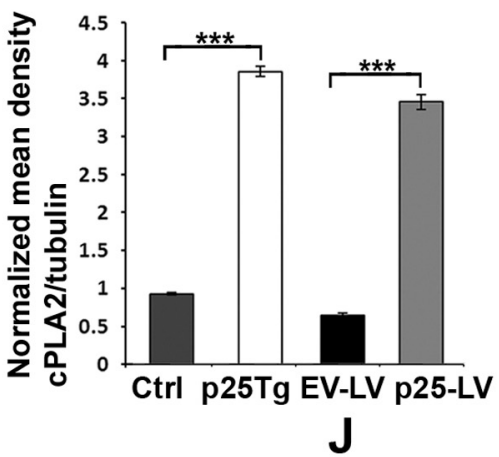

G
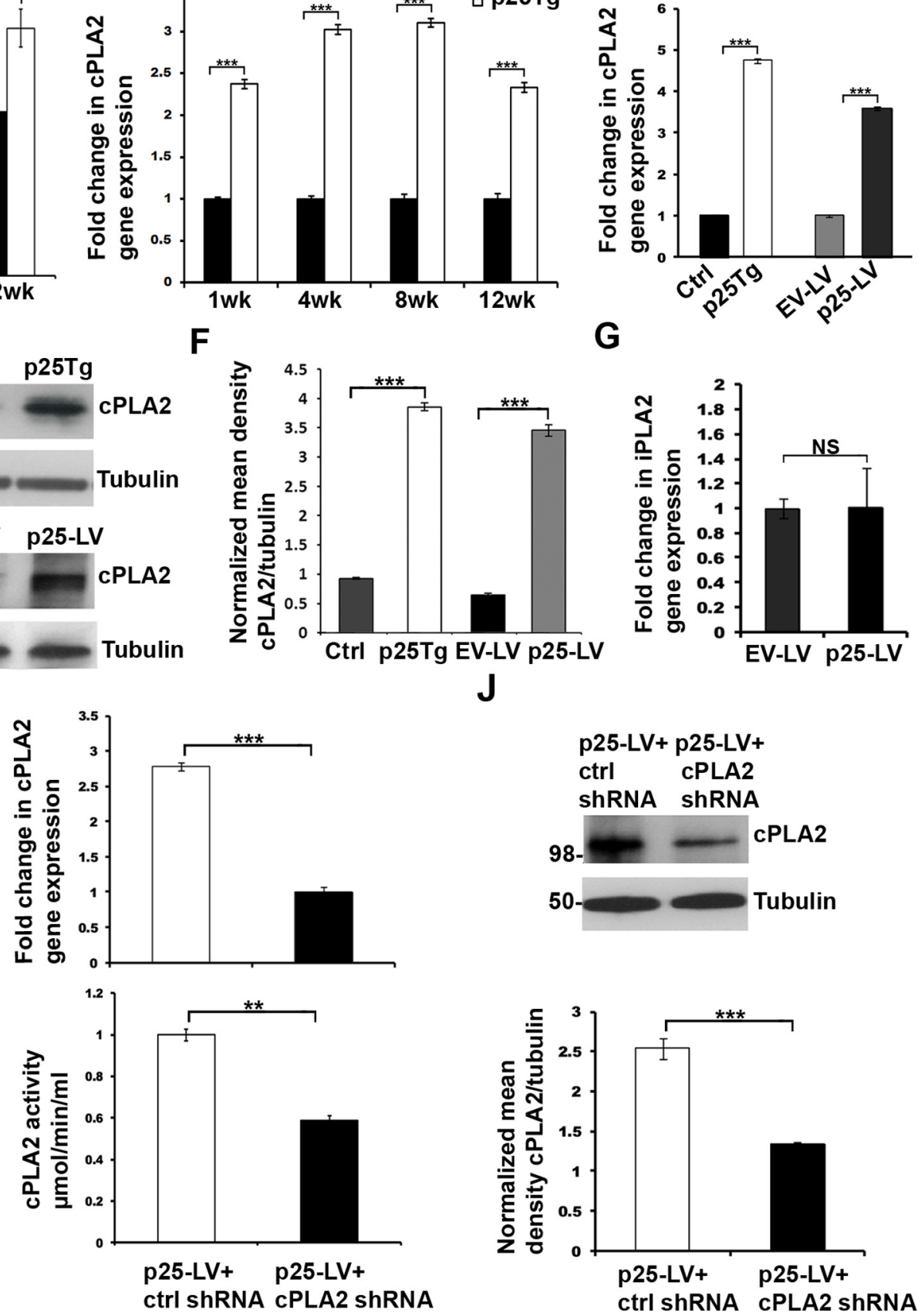

Figure 6. p25 expression causes an increase in CPLA2 expression and activity. $\boldsymbol{A}$, Mass spectrometric analyses results for the brain samples from p25Tg/control mice. Results were normalized against the internal standards of $\mathrm{LPC}\left({ }^{* *} p<0.01,{ }^{*} p<0.05 ; \mathrm{NS}, p>0.1\right)$. B, Quantitative real-time PCR results of CPLA2 gene expression in $1,4,8$, and 12 week induced $p 25 \mathrm{Tg}$ mice and age matched control mice $\left.{ }^{* * *} p<0.001\right)$.C, RT-PCR results of cPLA2 gene expression in neurons from p25Tg/control mice and neurons transduced with EV-LV/p25-LV (*** $\left.p<0.001\right) . D, c P L A 2$ activity assays were performed with lysates from neurons of $\mathrm{p} 25 \mathrm{Tg} / \mathrm{control}$ mice and neurons transduced with EV-LV/p25-LV (*** $<0.001)$. $\boldsymbol{E}$, Western blot analyses were performed on lysates from the samples same as in D using anti-cPLA2 antibody. F, Quantification of immunoblot analyses in $\boldsymbol{E}$ (*** $p 0.001)$. G, Real-time PCR results of iPLA2 gene expression in cortical neurons transduced with EV-LV/p25-LV (NS, $p>0.1)$. $\boldsymbol{H}$, Glia were treated with supernatants from cortical neurons transduced with EV-LV/p25-LV or p25-LV+ cPLA2 inhibitor (AACOCF3)/p25-LV+iPLA2 inhibitor (BEL). Immunocytochemistry was performed on the glia with anti-GFAP antibody (green) and DAPI (blue). Scale bars, $20 \mu \mathrm{m}$. I, RT-PCR results of CPLA2 gene expression in 7-DIC cortical neurons transduced with p25-LV + cPLA2 shRNA or p25-LV + control (ctrl) shRNA (*** $<0.001)$. Graph in the bottom panel shows the results of cPLA2 activity assays in neurons transduced with p25-LV + cPLA2 shRNA or p25-LV + control shRNA $\left.*^{* *} p<0.01\right)$. J, Western blot analyses were performed on samples from neurons transduced with p25-LV + cPLA2 shRNA or p25-LV + control shRNA using anti-cPLA2 antibody. Tubulin acts as a loading control for Western blot analyses. Quantification of immunoblots was shown in the bottom (*** $p<0.001)$. Error bars indicate $\pm S E M$.

However, an earlier study showed significant reduction of neurons after LPC injection (Sheikh et al., 2009). This differential observation could be due to the differences in the form of commercial LPC, injection site and susceptibilities of dif- ferent neuronal subtypes to a toxic insult. Together, our results show that an inflammatory component produced during the early event of p25-induced neuroinflammation triggers neuropathological changes found in AD. 
A

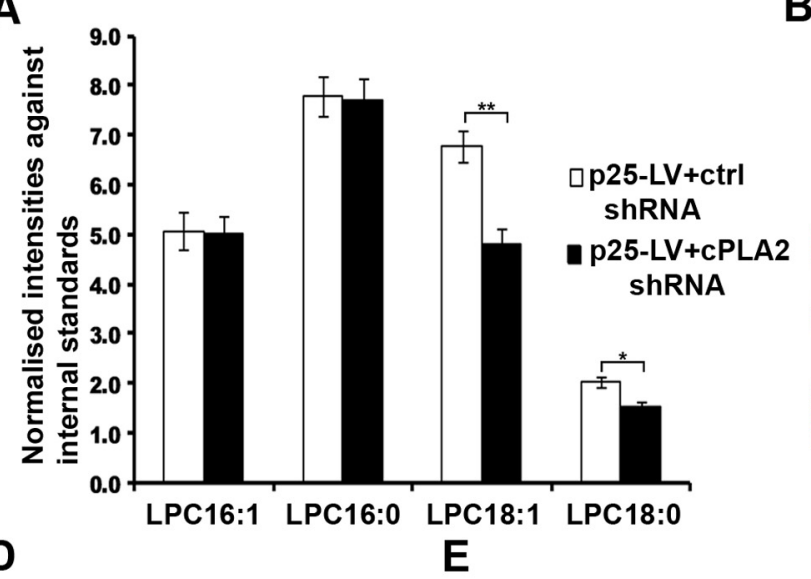

B

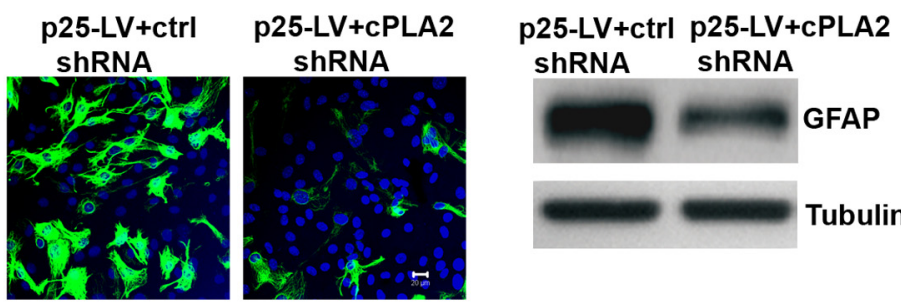

C

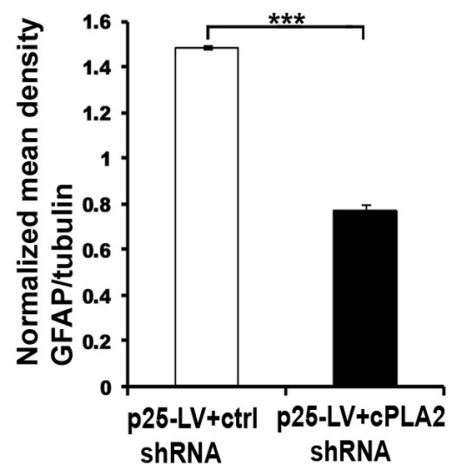

G

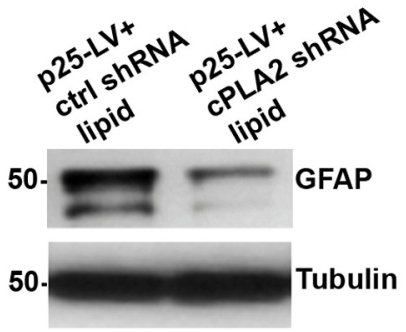

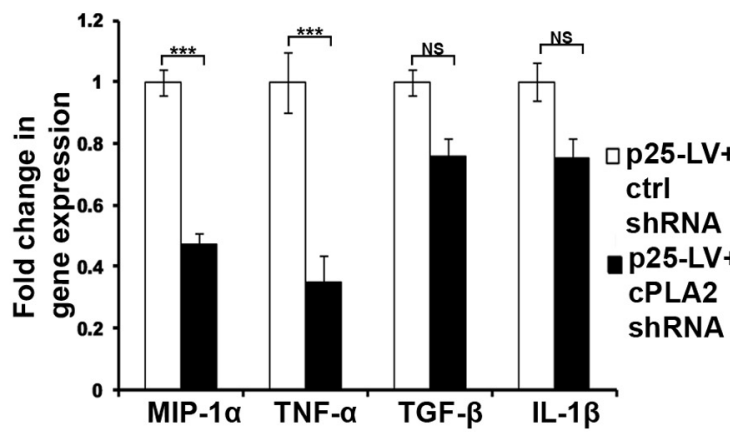

H

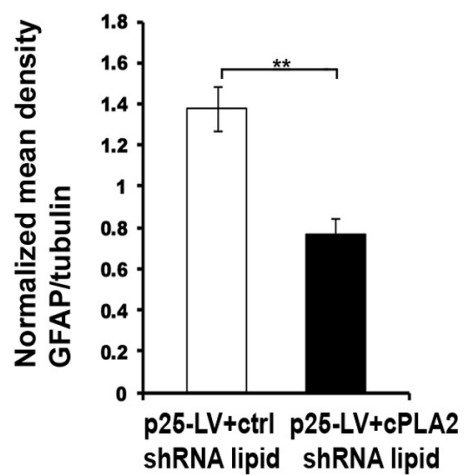


A

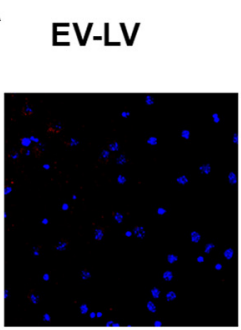

p25-LV+

p25-LV+ ctrl shRNA cPLA2 ShRNA
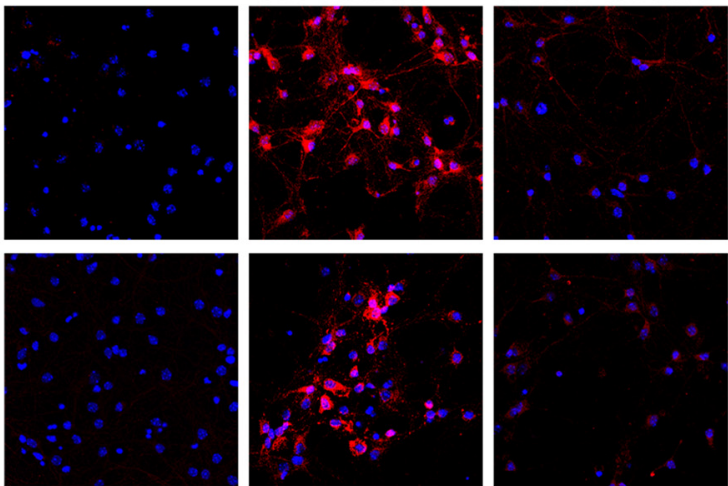

LPC

Vehicle
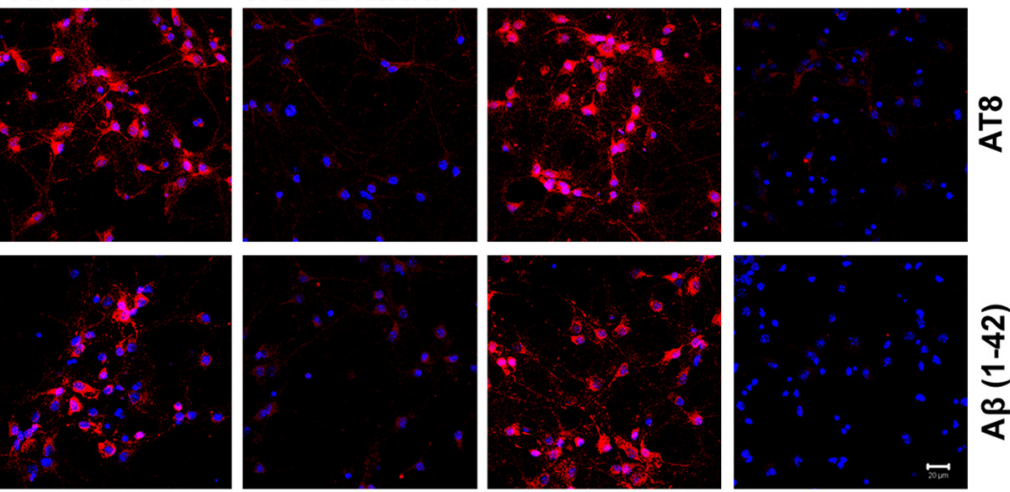

B

EV-LV

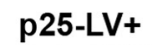
ctrl shRNA

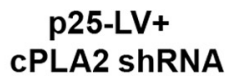

LPC
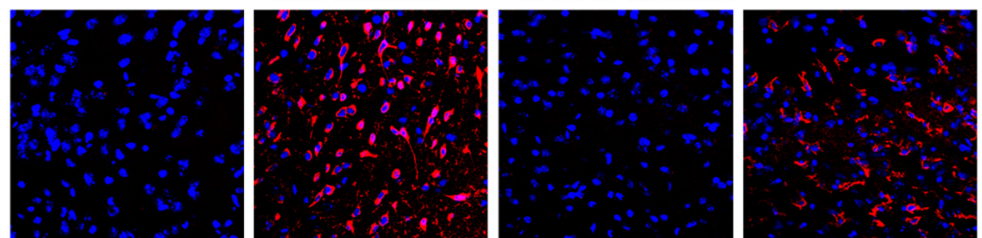

Vehicle
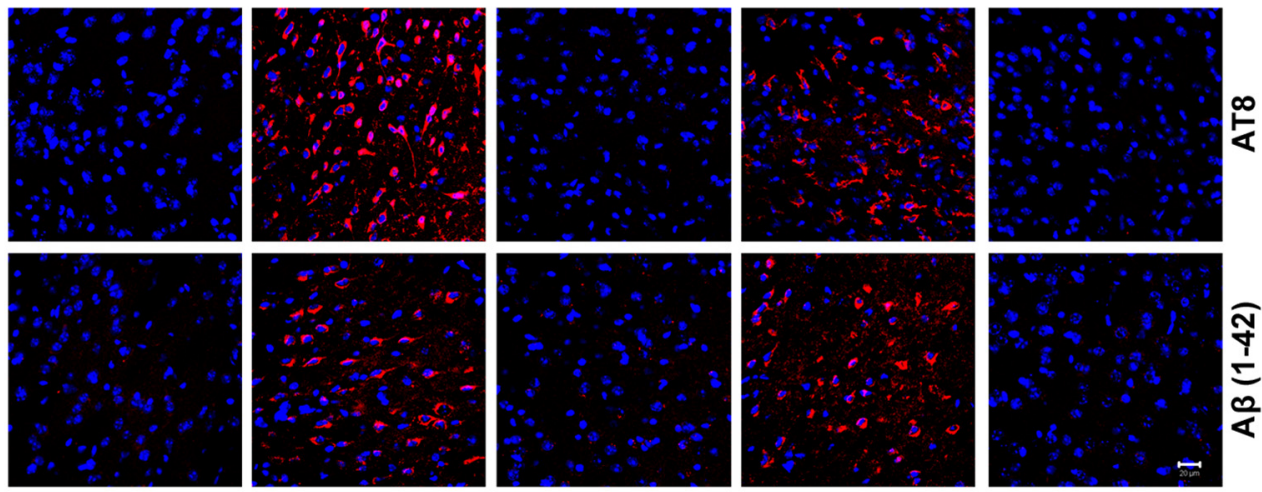

C

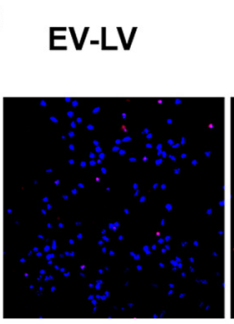

p25-LV+

p25-LV+

D ctrl shRNA
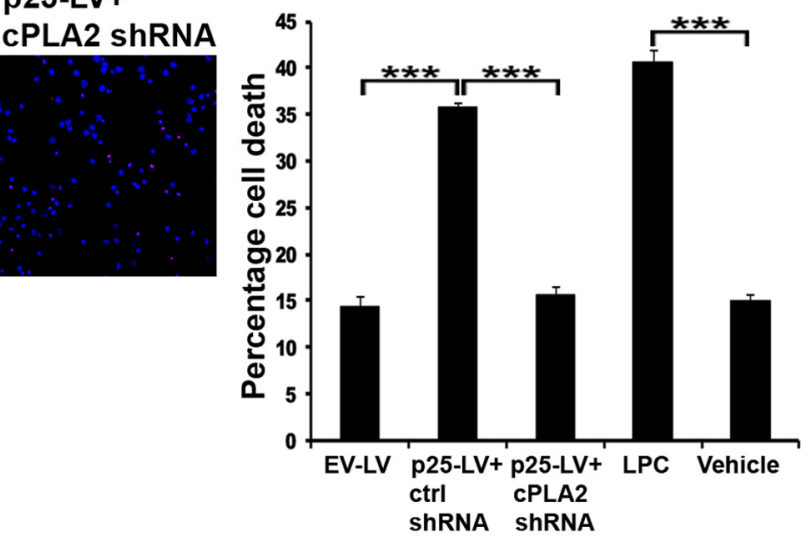

Figure 8. $\quad$ p25-mediated neuroinflammation is a trigger for neurodegeneration. $A$, In vitro treatment of 7-DIC cortical neurons with cell-free supernatants from glia that treated with lipids extracted from EV-LV, p25-LV + control shRNA and p25-LV + cPLA2 shRNA-transduced neurons and also from glia treated with LPC18:1/vehicle (chloroform/Methanol) for $48 \mathrm{~h}$. The neurons were then fixed and immunostained with antibodies specific to phospho-tau (AT8) and A $\beta 1-42$. Nuclei were counterstained with DAPI. Scale bars, $20 \mu \mathrm{m}$. B, In vivo injections of LPC18:1, vehicle and lipids extracted from EV-LV, p25-LV + control shRNA and p25-LV + cPLA2 shRNA-transduced neurons into mice brain. The animals were perfused after $4 \mathrm{~d}$ and the brain sections were immunostained with antibodies specific to phospho-tau and A $\beta$ 1-42 (red). Nuclei were counterstained with DAPI (blue). Scale bars, $20 \mu \mathrm{m}$. C, TUNEL staining of cortical neurons incubated with supernatants from glia treated with vehicle, LPC 18:1 and lipids extracted from EV-LV, p25-LV + control shRNA and p25-LV + cPLA2 shRNA-treated neurons. Scale bars, $20 \mu \mathrm{m}$. D, Percentage cell death in $C$ was calculated by counting the TUNEL-positive cells normalized with DAPI from 10 independent fields (*** $p<0.001)$. Error bars indicate \pm SEM.

al., 2008), this is the first report to provide a link between the two processes using in vivo $\mathrm{p} 25 \mathrm{Tg}$ mice and in vitro $\mathrm{p} 25$ overexpressing neurons as well as identifying a specific lipid factor responsible for triggering one phase of the neurodegenerative process. We determined that neuroinflammation is an early event in p25Tg mice, where marked increases in GFAP and proinflammatory cytokines levels in the brain were observed even after 1 week of induction. However, microgliosis was not observed at this time point and was evident only during later induction periods in p25Tg mice. In contrast, we did not observe any microglial activation in our mixed glia culture treated either with the conditioned media or with the lipid extracts from the p 25 overexpressed neurons for $48 \mathrm{~h}$ (data not shown). This might possibly be because of the less abundance of microglia in our in vitro system compared with in vivo. 
Moreover, conditioned media transfer experiments between microglia and p25 overexpressed neurons did not show any significant changes in microglial activation (data not shown). Our finding of astrogliosis preceding microgliosis in p25mediated neuroinflammation is also supported by various other studies where chemokines released by astrocytes attract microglia which further express proinflammatory products contributing to the additional damage to neurons (Hurwitz et al., 1995; Tuppo and Arias, 2005). Neuroinflammation has been previously reported to be initiated by amyloid formation in various neurodegenerative models (Combs et al., 2001; White et al., 2005). However, our data suggest an alternate trigger for astrogliosis that occurs even before any evidence of amyloid pathology in p25Tg mice which becomes evident after 8 weeks of p25 expression. Our finding is also supported by studies where elevations of GFAP were not caused by the consequence of amyloid accumulation (Ingelsson et al., 2004; Zhu et al., 2008) and the data presented in this report suggests that the alternate pathway could be p25-mediated LPC production. Although, the involvement of CD4+ and $\mathrm{CD} 8+\mathrm{T}$ cell recruitment in neuroinflammation has been previously reported in neurodegenerative diseases (Brisebois et al., 2006; Brochard et al., 2009), this is the first report showing that p25 overexpression may initiate the peripheral cell recruitment into the mice brain to exacerbate neuroinflammation. LPC has previously been shown to be a chemoattractant for T cells (Ousman and David, 2000; Zhang et al., 2007), therefore it is reasonable to believe that p25-mediated LPC production is responsible for this peripheral cell recruitment.

To gain insight into the mechanism of p25-mediated neuroinflammation, we performed coculture as well as conditioned media transfer experiments to identify the soluble factor that is released from p25 expressing neurons to activate astrocytes. We then elucidated the nature of the soluble signal by factor removal experiments using mass spectrometry lipidomics to identify that the lipid was lysophosphatidylcholine (LPC). Our findings have been supported by previous studies where, increased LPC levels have been observed in multiple sclerosis and in aged human brain (Andreoli et al., 1973; Wender et al., 1988). However, the precise mechanism behind LPC production has not been fully elucidated. We characterized the particular subtype of LPC responsible for astrogliosis as LPC 18.1. Previous studies suggest that the potency of LPC may vary based on the relative length, position, and unsaturation of acyl chain. The potency of LPC is also determined by the ability to form the active micelles and the unsaturated LPC (18:1) could readily form the active micelles compared with the other saturated isoforms (Lauber et al., 2003; Ojala et al., 2007).

LPC is produced by the hydrolysis of phosphatidylcholine via the action of phospholipase A2 (PLA2) (Steinbrecher et al.,
1984). Recent studies suggest that increased PLA2 activity and PLA2-generated proinflammatory mediators play a central role in inflammatory responses associated with neurological disorders such as ischemia, AD, PD, and MS (Farooqui et al., 2006). To date, cytosolic PLA2 (cPLA2-IV), $\mathrm{Ca}^{2+}$-dependent PLA2 (iPLA2-VI) and secretory PLA2 (sPLA2-II) are the major PLA2 types that were involved in inflammatory-mediated neurodegeneration. Although, sPLA2 isoforms X, V and III produce LPC, the neuroinflammatory role of these isoforms has not been elucidated (Dennis, 1997; Sun et al., 2004). The isoform sPLA2-II is absent in our mouse model and primary neuronal cultures because of an inbred gene deletion in the C57BL/6 mice strain (Kennedy et al., 1995). The isoform iPLA2 is generally observed as a housekeeping enzyme for the maintenance of membrane phospholipids (Balsinde and Dennis, 1997). Our qRT-PCR and PLA2 inhibitor studies using AACOCF3 (cPLA2 inhibitor) and BEL (iPLA2 inhibitor) in the p25 overexpressing neurons, confirm that $\mathrm{p} 25$ overexpression does not affect iPLA2 levels. AACOCF3 is 500 -fold more potent inhibitor of cPLA2 than sPLA2. AACOCF3 inhibits bovine brain CPLA2 and IPLA2 in a dosedependent manner with IC50 values of 1.5 and $6.0 \mu \mathrm{M}$, respectively. BEL is $>1,000$-fold selective for iPLA2 than cPLA2 (Riendeau et al., 1994; Jenkins et al., 2002; Farooqui et al., 2006). 
We show that p25 overexpression increases LPC levels with a concomitant upregulation of cPLA2 expression and activity in all the p25 overexpressing systems including p25Tg mice, primary neurons from the $\mathrm{p} 25 \mathrm{Tg}$ mice and lentiviral-transduced neurons. Increased cPLA2 activity has been observed in AD brains in association with A $\beta$ toxicity (Stephenson et al., 1996; Farooqui et al., 1997; Kriem et al., 2005). However, our studies in p25Tg mice show that upregulation of cPLA2 occurred before any phosphotau and amyloid formation (Figs. 2A, 6B). Overexpression of p25 may mediate this response via the transcriptional regulation of cPLA2 gene expression, since p25/Cdk5 activity has been observed in the nucleus of neurons (Patrick et al., 1999). To investigate whether the reduction of p25-mediated cPLA2 upregulation could ameliorate the pathological progression, we silenced CPLA2 gene expression and found dramatic reductions in LPC 18:1 and significant decreases in the expression of neuroinflammatory markers (Fig. 7). To delineate whether the p25 induced inflammatory mediators production can have the ability to trigger neurodegeneration, we performed conditional media transfer experiments between the activated glia and healthy cortical neurons. Our results suggest that p25-induced neuroinflammation may be an initial trigger for neurodegeneration. However, absence of TUNEL positive cells in the in vivo studies suggests that there is a possibility of involvement other pathways in the progression of neurodegeneration. Marked reductions in neuropathological markers expression in experiments with lipid from cPLA2 silenced p25 overexpressing neurons further confirm our findings. Together, our results indicate that cPLA2 is upregulated when p 25 is produced, resulting in subsequent neuroinflammation and neurodegeneration. Recent studies support our findings where, CDP-choline (a cPLA2 inhibitor) protected neurons from glutamate-mediated neurotoxicity (Mir et al., 2003).

We propose that Cdk5/p25 hyperactivation causes cPLA2 upregulation and releases soluble LPC which activates glia to produce proinflammatory chemokines and cytokines. LPC and subsequent inflammatory mediator also triggers recruitment of peripheral immune cells into the brain, which could then initiate another phase of neuroinflammatory changes in the CNS causing further damage. Finally, the inflammatory mediators trigger the initiation of AD-like pathological outcomes (Fig. 9).

There is no optimal approach to date supporting the treatment of $\mathrm{AD}$ patients with anti-inflammatory drugs (Imbimbo, 2009) and it would be interesting to investigate the effect of specific inhibition of cPLA2 in neurodegenerative disease progression. Specific knock down of cPLA2 in p25Tg mice would be an interesting experiment to investigate $\mathrm{p}$-tau, $\mathrm{A} \beta \mathrm{1}-42$ and neuronal death in these mice especially in the absence of tau and amyloid pathology early in the disease process. However, knockdown of cPLA2 using cPLA2 shRNA lentivirus in p25Tg mice would be challenging due to the sustained inflammatory trigger by constant p25 expression and cPLA2 upregulation in p25Tg mice. Repeated injections with shRNA virus would be required to get the optimal result which is detrimental to the mice. Crossing cPLA2 knock-out mice with p25Tg mice would be another possible study to further expand knowledge of the mechanisms in inflammatory-mediated neurodegenerative disease progression.

In conclusion, we demonstrate for the first time that LPC is a molecular signal released from neurons to mediate glial activation and the recruitment of inflammatory mediators in $\mathrm{p} 25 \mathrm{Tg}$ mice contributing to AD-like neuropathology. We also identify cPLA2 and the specific LPC subtype responsible for the pathological effects in the brain. The identification of a specific inhibitor of cPLA2 in humans could therefore be a viable therapeutic intervention to treat neuroinflammation in neurodegenerative diseases.

\section{References}

Andreoli VM, Maffei F, Tonon GC, Zibetti A (1973) Significance of plasma lysolecithin in patients with multiple sclerosis: a longitudinal study. J Neurol Neurosurg Psychiatry 36:661-667.

Balsinde J, Dennis EA (1997) Function and inhibition of intracellular calcium-independent phospholipase A2. J Biol Chem 272:16069-16072.

Bian F, Nath R, Sobocinski G, Booher RN, Lipinski WJ, Callahan MJ, Pack A, Wang KK, Walker LC (2002) Axonopathy, tau abnormalities, and dyskinesia, but no neurofibrillary tangles in p25-transgenic mice. J Comp Neurol 446:257-266.

Bremer J, Norum KR (1982) Metabolism of very long-chain monounsaturated fatty acids (22:1) and the adaptation to their presence in the diet. J Lipid Res 23:243-256.

Brisebois M, Zehntner SP, Estrada J, Owens T, Fournier S (2006) A pathogenic role for $\mathrm{CD} 8+\mathrm{T}$ cells in a spontaneous model of demyelinating disease. J Immunol 177:2403-2411.

Brochard V, Combadière B, Prigent A, Laouar Y, Perrin A, Beray-Berthat V, Bonduelle O, Alvarez-Fischer D, Callebert J, Launay JM, Duyckaerts C, Flavell RA, Hirsch EC, Hunot S (2009) Infiltration of CD4+ lymphocytes into the brain contributes to neurodegeneration in a mouse model of Parkinson disease. J Clin Invest 119:182-192.

Chan R, Uchil PD, Jin J, Shui G, Ott DE, Mothes W, Wenk MR (2008) Retroviruses human immunodeficiency virus and murine leukemia virus are enriched in phosphoinositides. J Virol 82:11228-11238.

Combs CK, Karlo JC, Kao SC, Landreth GE (2001) beta-Amyloid stimulation of microglia and monocytes results in TNFalpha-dependent expression of inducible nitric oxide synthase and neuronal apoptosis. J Neurosci 21:1179-1188.

Cruz JC, Tseng HC, Goldman JA, Shih H, Tsai LH (2003) Aberrant Cdk5 activation by $\mathrm{p} 25$ triggers pathological events leading to neurodegeneration and neurofibrillary tangles. Neuron 40:471-483.

Dennis EA (1997) The growing phospholipase A2 superfamily of signal transduction enzymes. Trends Biochem Sci 22:1-2.

Farooqui AA, Rapoport SI, Horrocks LA (1997) Membrane phospholipid alterations in Alzheimer's disease: deficiency of ethanolamine plasmalogens. Neurochem Res 22:523-527.

Farooqui AA, Ong WY, Horrocks LA (2006) Inhibitors of brain phospholipase A2 activity: their neuropharmacological effects and therapeutic importance for the treatment of neurologic disorders. Pharmacol Rev 58:591-620.

Hallows JL, Iosif RE, Biasell RD, Vincent I (2006) p35/p25 is not essential for tau and cytoskeletal pathology or neuronal loss in Niemann-Pick type C disease. J Neurosci 26:2738-2744.

Hurwitz AA, Lyman WD, Berman JW (1995) Tumor necrosis factor alpha and transforming growth factor beta upregulate astrocyte expression of monocyte chemoattractant protein-1. J Neuroimmunol 57:193-198.

Imbimbo BP (2009) An update on the efficacy of non-steroidal antiinflammatory drugs in Alzheimer's disease. Expert Opin Investig Drugs 18:1147-1168.

Ingelsson $\mathrm{M}$, Fukumoto $\mathrm{H}$, Newell KL, Growdon JH, Hedley-Whyte ET, Frosch MP, Albert MS, Hyman BT, Irizarry MC (2004) Early Abeta accumulation and progressive synaptic loss, gliosis, and tangle formation in AD brain. Neurology 62:925-931.

Jenkins CM, Han X, Mancuso DJ, Gross RW (2002) Identification of calciumindependent phospholipase A2 (iPLA2) beta, and not iPLA2gamma, as the mediator of arginine vasopressin-induced arachidonic acid release in A-10 smooth muscle cells. Enantioselective mechanism-based discrimination of mammalian iPLA2s. J Biol Chem 277:32807-32814.

Keller JN, Hanni KB, Markesbery WR (2000) Possible involvement of proteasome inhibition in aging: implications for oxidative stress. Mech Ageing Dev 113:61-70.

Kennedy BP, Payette P, Mudgett J, Vadas P, Pruzanski W, Kwan M, Tang C, Rancourt DE, Cromlish WA (1995) A natural disruption of the secretory group II phospholipase A2 gene in inbred mouse strains. J Biol Chem 270:22378-22385.

Kesavapany S, Li BS, Amin N, Zheng YL, Grant P, Pant HC (2004a) Neuronal cyclin-dependent kinase 5: role in nervous system function and its specific inhibition by the Cdk5 inhibitory peptide. Biochim Biophys Acta 1697:143-153. 
Kesavapany S, Amin N, Zheng YL, Nijhara R, Jaffe H, Sihag R, Gutkind JS, Takahashi S, Kulkarni A, Grant P, Pant HC (2004b) p35/cyclindependent kinase 5 phosphorylation of ras guanine nucleotide releasing factor 2 (RasGRF2) mediates Rac-dependent Extracellular Signalregulated kinase 1/2 activity, altering RasGRF2 and microtubuleassociated protein 1b distribution in neurons. J Neurosci 24:4421-4431.

Kitazawa M, Oddo S, Yamasaki TR, Green KN, LaFerla FM (2005) Lipopolysaccharide-induced inflammation exacerbates tau pathology by a cyclin-dependent kinase 5-mediated pathway in a transgenic model of Alzheimer's disease. J Neurosci 25:8843-8853.

Komatsu M, Waguri S, Chiba T, Murata S, Iwata J, Tanida I, Ueno T, Koike M, Uchiyama Y, Kominami E, Tanaka K (2006) Loss of autophagy in the central nervous system causes neurodegeneration in mice. Nature 441:880-884.

Kriem B, Sponne I, Fifre A, Malaplate-Armand C, Lozac'h-Pillot K, Koziel V, Yen-Potin FT, Bihain B, Oster T, Olivier JL, Pillot T (2005) Cytosolic phospholipase A2 mediates neuronal apoptosis induced by soluble oligomers of the amyloid-beta peptide. FASEB J 19:85-87.

Lauber K, Bohn E, Kröber SM, Xiao YJ, Blumenthal SG, Lindemann RK, Marini P, Wiedig C, Zobywalski A, Baksh S, Xu Y, Autenrieth IB, SchulzeOsthoff K, Belka C, Stuhler G, Wesselborg S (2003) Apoptotic cells induce migration of phagocytes via caspase-3-mediated release of a lipid attraction signal. Cell 113:717-730.

Lopes JP, Oliveira CR, Agostinho P (2007) Role of cyclin-dependent kinase 5 in the neurodegenerative process triggered by amyloid-Beta and prion peptides: implications for Alzheimer's disease and prion-related encephalopathies. Cell Mol Neurobiol 27:943-957.

Markiewicz I, Lukomska B (2006) The role of astrocytes in the physiology and pathology of the central nervous system. Acta Neurobiol Exp (Wars) 66:343-358

Matsuoka Y, Picciano M, Malester B, LaFrancois J, Zehr C, Daeschner JM, Olschowka JA, Fonseca MI, O’Banion MK, Tenner AJ, Lemere CA, Duff K (2001) Inflammatory responses to amyloidosis in a transgenic mouse model of Alzheimer's disease. Am J Pathol 158:1345-1354.

Meyerson M, Faha B, Su LK, Harlow E, Tsai LH (1991) The cyclindependent kinase family. Cold Spring Harb Symp Quant Biol 56:177-186.

Mir C, Clotet J, Aledo R, Durany N, Argemí J, Lozano R, Cervós-Navarro J, Casals N (2003) CDP-choline prevents glutamate-mediated cell death in cerebellar granule neurons. J Mol Neurosci 20:53-60.

Muyllaert D, Terwel D, Kremer A, Sennvik K, Borghgraef P, Devijver H, Dewachter I, Van Leuven F (2008) Neurodegeneration and neuroinflammation in cdk5/p25-inducible mice: a model for hippocampal sclerosis and neocortical degeneration. Am J Pathol 172:470-485.

Nguyen MD, Larivière RC, Julien JP (2001) Deregulation of Cdk5 in a mouse model of ALS: toxicity alleviated by perikaryal neurofilament inclusions. Neuron 30:135-147.

Noble W, Olm V, Takata K, Casey E, Mary O, Meyerson J, Gaynor K, LaFrancois J, Wang L, Kondo T, Davies P, Burns M, Veeranna, Nixon R, Dickson D, Matsuoka Y, Ahlijanian M, Lau LF, Duff K (2003) Cdk5 is a key factor in tau aggregation and tangle formation in vivo. Neuron 38:555-565.

Ojala PJ, Hirvonen TE, Hermansson M, Somerharju P, Parkkinen J (2007) Acyl chain-dependent effect of lysophosphatidylcholine on human neutrophils. J Leukoc Biol 82:1501-1509.

Otth C, Concha II, Arendt T, Stieler J, Schliebs R, González-Billault C, Maccioni RB (2002) AbetaPP induces cdk5-dependent tau hyperphosphorylation in transgenic mice Tg2576. J Alzheimers Dis 4:417-430.

Ousman SS, David S (2000) Lysophosphatidylcholine induces rapid recruitment and activation of macrophages in the adult mouse spinal cord. Glia 30:92-104.

Patrick GN, Zukerberg L, Nikolic M, de la Monte S, Dikkes P, Tsai LH (1999) Conversion of $\mathrm{p} 35$ to $\mathrm{p} 25$ deregulates $\mathrm{Cdk} 5$ activity and promotes neurodegeneration. Nature 402:615-622.

Poore CP, Sundaram JR, Pareek TK, Fu A, Amin N, Mohamed NE, Zheng YL, Goh AX, Lai MK, Ip NY, Pant HC, Kesavapany S (2010) Cdk5-mediated phosphorylation of delta-catenin regulates its localization and GluR2mediated synaptic activity. J Neurosci 30:8457-8467.
Riendeau D, Guay J, Weech PK, Laliberté F, Yergey J, Li C, Desmarais S, Perrier H, Liu S, Nicoll-Griffith D, et al. (1994) Arachidonyl trifluoromethyl ketone, a potent inhibitor of $85-\mathrm{kDa}$ phospholipase A2, blocks production of arachidonate and 12-hydroxyeicosatetraenoic acid by calcium ionophore-challenged platelets. J Biol Chem 269:15619-15624.

Saito T, Konno T, Hosokawa T, Asada A, Ishiguro K, Hisanaga S (2007) p25/cyclin-dependent kinase 5 promotes the progression of cell death in nucleus of endoplasmic reticulum-stressed neurons. J Neurochem 102:133-140.

Sheikh AM, Nagai A, Ryu JK, McLarnon JG, Kim SU, Masuda J (2009) Lysophosphatidylcholine induces glial cell activation: role of rho kinase. Glia 57:898-907.

Shui G, Guan XL, Gopalakrishnan P, Xue Y, Goh JS, Yang H, Wenk MR (2010) Characterization of substrate preference for Slclp and Cst26p in Saccharomyces cerevisiae using lipidomic approaches and an LPAAT activity assay. PLoS One 5:e11956.

Smith PD, Mount MP, Shree R, Callaghan S, Slack RS, Anisman H, Vincent I, Wang X, Mao Z, Park DS (2006) Calpain-regulated p35/cdk5 plays a central role in dopaminergic neuron death through modulation of the transcription factor myocyte enhancer factor 2. J Neurosci 26:440-447.

Steinbrecher UP, Parthasarathy S, Leake DS, Witztum JL, Steinberg D (1984) Modification of low density lipoprotein by endothelial cells involves lipid peroxidation and degradation of low density lipoprotein phospholipids. Proc Natl Acad Sci U S A 81:3883-3887.

Stephenson DT, Lemere CA, Selkoe DJ, Clemens JA (1996) Cytosolic phospholipase A2 (cPLA2) immunoreactivity is elevated in Alzheimer's disease brain. Neurobiol Dis 3:51-63.

Sun GY, Xu J, Jensen MD, Simonyi A (2004) Phospholipase A2 in the central nervous system: implications for neurodegenerative diseases. J Lipid Res 45:205-213.

Takahashi S, Ohshima T, Hirasawa M, Pareek TK, Bugge TH, Morozov A, Fujieda K, Brady RO, Kulkarni AB (2010) Conditional deletion of neuronal cyclin-dependent kinase 5 in developing forebrain results in microglial activation and neurodegeneration. Am J Pathol 176:320-329.

Takashima A, Murayama M, Yasutake K, Takahashi H, Yokoyama M, Ishiguro K (2001) Involvement of cyclin dependent kinase5 activator p25 on tau phosphorylation in mouse brain. Neurosci Lett 306:37-40.

Town T, Zolton J, Shaffner R, Schnell B, Crescentini R, Wu Y, Zeng J, DelleDonne A, Obregon D, Tan J, Mullan M (2002) p35/Cdk5 pathway mediates soluble amyloid-beta peptide-induced tau phosphorylation in vitro. J Neurosci Res 69:362-372.

Trojanowski JQ, Mawal-Dewan M, Schmidt ML, Martin J, Lee VM (1993) Localization of the mitogen activated protein kinase ERK2 in Alzheimer's disease neurofibrillary tangles and senile plaque neurites. Brain Res 618:333-337.

Tseng HC, Zhou Y, Shen Y, Tsai LH (2002) A survey of Cdk5 activator p35 and p25 levels in Alzheimer's disease brains. FEBS Lett 523:58-62.

Tuppo EE, Arias HR (2005) The role of inflammation in Alzheimer's disease. Int J Biochem Cell Biol 37:289-305.

Wender M, Adamczewska-Goncerzewicz Z, Szczech J, Godlewski A (1988) Myelin lipids in aging human brain. Neurochem Pathol 8:121-130.

White JA, Manelli AM, Holmberg KH, Van Eldik LJ, Ladu MJ (2005) Differential effects of oligomeric and fibrillar amyloid-beta 1-42 on astrocyte-mediated inflammation. Neurobiol Dis 18:459-465.

Zhang Z, Lee YC, Kim SJ, Choi MS, Tsai PC, Saha A, Wei H, Xu Y, Xiao YJ, Zhang P, Heffer A, Mukherjee AB (2007) Production of lysophosphatidylcholine by cPLA2 in the brain of mice lacking PPT1 is a signal for phagocyte infiltration. Hum Mol Genet 16:837-847.

Zheng YL, Kesavapany S, Gravell M, Hamilton RS, Schubert M, Amin N, Albers W, Grant P, Pant HC (2005) A Cdk5 inhibitory peptide reduces tau hyperphosphorylation and apoptosis in neurons. EMBO J 24:209-220.

Zhu M, Gu F, Shi J, Hu J, Hu Y, Zhao Z (2008) Increased oxidative stress and astrogliosis responses in conditional double-knockout mice of Alzheimer-like presenilin-1 and presenilin-2. Free Radic Biol Med 45: 1493-1499. 